\title{
Formal Modeling and Verification of Concurrent FSMs: Case Study on Event-Based Cooperative Transport Robots
}

\author{
Yoshinao ISOBE $^{\dagger \text { a) }}$, Member, Nobuhiko MIYAMOTO ${ }^{\dagger}$, Noriaki $\mathrm{ANDO}^{\dagger}$, and Yutaka OIWA ${ }^{\dagger}$, Nonmembers
}

SUMMARY In this paper, we demonstrate that a formal approach is effective for improving reliability of cooperative robot designs, where the control logics are expressed in concurrent FSMs (Finite State Machines), especially in accordance with the standard FSM4RTC (FSM for Robotic Technology Components), by a case study of cooperative transport robots. In the case study, FSMs are modeled in the formal specification language CSP (Communicating Sequential Processes) and checked by the modelchecking tool FDR, where we show techniques for modeling and verification of cooperative robots implemented with the help of the RTM (Robotic Technology Middleware).

key words: formal approach, concurrent finite state machines, process algebra, model check, cooperative robots, robotic technology middleware

\section{Introduction}

Event-based cooperative robot systems with discrete dynamics, such that a number of robots with several control modes cooperatively work on complex tasks by exchanging events (messages), can increase the robustness and the parallelism of work and can reduce the frequency of communications between robots. However, the design of the control software for such robot systems is often difficult because the whole behavior of the robot systems is the result of the interactions between discrete transitions in distributed (decentralized) autonomous robots.

Various formal approaches have been applied to verification of autonomous robotic systems, as shown in the survey by Luckcuck et al. [1]. For example, model-checkers are useful tools for automatically verifying complex interactive behaviors, but the state-space explosion problem still often makes it difficult to apply them to practical systems because the number of states of cooperative systems exponentially increases with the number of robot components. Therefore, the comprehensive formal approach for cooperative systems has not been established yet.

In this paper, we report a case study on formalization and verification of a design for cooperative transport robots, where two mobile robots cooperatively transport a box and each robot has five control-modes designed by finite state machines (FSMs). The robots sparsely communicate with each other by exchanging event-messages once a few seconds on average only when they change their control modes.

\footnotetext{
Manuscript received November 9, 2020.

Manuscript revised March 4, 2021.

Manuscript publicized July 8, 2021.

${ }^{\dagger}$ The authors are with National Institute of Advanced Industrial Science and Technology, Ikeda-shi, 563-8577 Japan.

a) E-mail: y-isobe@aist.go.jp

DOI: 10.1587/transinf.2020FOP0002
}

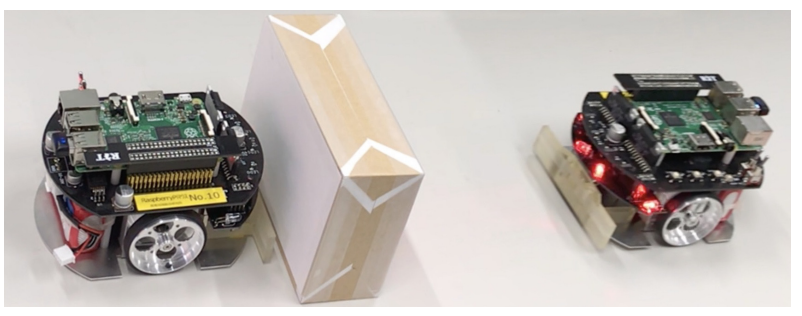

Fig. 1 The cooperative transport robots by Raspberry-Pi Mouse

They have no shared memory and there is no centralized controller. In this case, each event-message is very important and must be carefully designed. Therefore, we formally describe the robot in the specification language CSP (Communicating Sequential Processes) [2]-[4] and then we exhaustively verify the cooperative behavior by the model checker FDR (Failures-Divergences Refinement) [5], where we focus on the verification of discrete dynamics (e.g. notifications of mode-changes) rather than continuous dynamics (e.g. control of speeds or directions). We also give reusable formal descriptions for connecting robot-components and for verifying the behavior.

Furthermore, we report an implementation of the formally designed cooperative transport robots. For demonstrating how the robots cooperatively behave in the real world, we use Raspberry-Pi Mouse [6], [7], which is a wheel-type mobile robot equipped with a small singleboard computer Raspberry-Pi as shown in Fig. 1, and we use RT-Middleware (RTM) [8]-[10] with the help of FSM4RTC [11], where RT stands for "Robotic Technology" and FSM4RTC stands for "Finite State Machines (FSMs) for RT-Components (RTCs)". RTM is a software platform standard in OMG (Object Management Group) for constructing robot systems by hierarchically connecting robotic software components called RTC [12]. FSM4RTC [13] is an extended-standard of RTC in OMG for supporting implementation of FSMs in RTCs. The RTC software for Raspberry-Pi Mouse can be installed from the GitHub [7].

The contribution of this paper is summarized as follows:

- We show a formal development-process for cooperative robots with discrete dynamics as follows: design by concurrent FSM, formalization in CSP, verification by FDR, and implementation on RTM with FSM4RTC, as shown in Fig. 2.

- We demonstrate how to formalize cooperative robots 


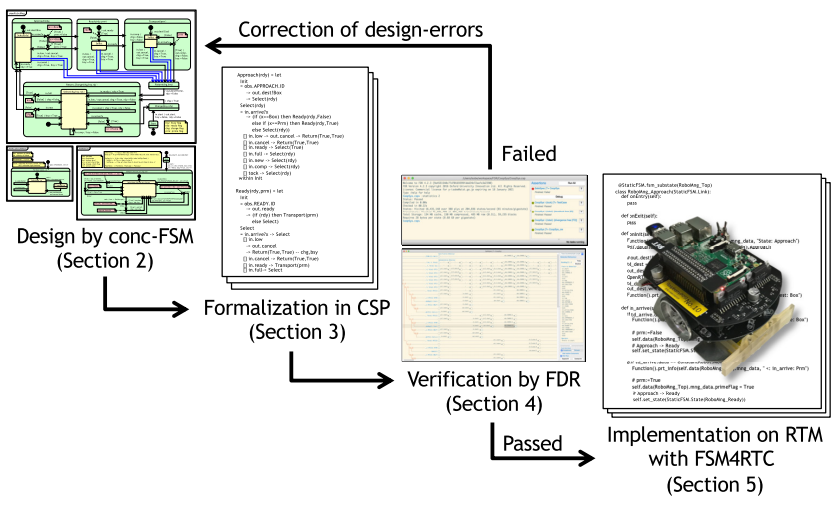

Fig. 2 The formal development-process for cooperative robots

and verify them in detail, where some formal descriptions are generic and reusable for connecting RTCs and expressing requirements.

This paper is organized as follows: At first, in Sect. 2, we introduce an example of cooperative transport robots, which is used as a case study in this paper, and give finite state machines (FSMs) of the components in the robots. Next, in Sects. 3 and 4, we formalize the behaviors of the robots and verify them, respectively. Then, in Sect. 5, we briefly explain the implementation of the robots in RTM. Finally, in Sects. 6 and 7, we review related works and conclude this paper.

\section{An Example: Cooperative Transport Robots}

In this section, we explain a cooperative robot system, named Coopsys, used for a case study in this paper. The basic idea of CoopSys is similar to the cooperative robots which is formally described in hybrid automata (but is not formally verified) by Chaimowicz et al. [14], but the details, e.g. the structure of the robot, control-modes, and event-messages, are different. For example, in CoopSys, each robot consists of a abstract (i.e. high-level) management component for interaction with the other robots and a hardware-dependent (i.e. low-level) control component for movement, so that the management component can be reused for the other robot-hardware.

At first, in Sect. 2.1, we briefly introduce the cooperative robot system CoopSys. Next, in Sect. 2.2, we explain the structure of CoopSys as a concurrent composition of RTCs (RT-Components), and then in Sect. 2.3, we give the finite state machines FSMs of the RTCs in CoopSys.

\subsection{Introduction to CoopSys}

As shown in the top of Fig. 3, the cooperative robot system consists of two cooperative robots CoopRobo(1), (2), two home bases (charging stations) Home ${ }_{1,2}$, the goal location Goal, and an object box Box. The two robots cooperatively transport the box to the goal location, where each robot has a rechargeable battery and can charge on its home base. Here,

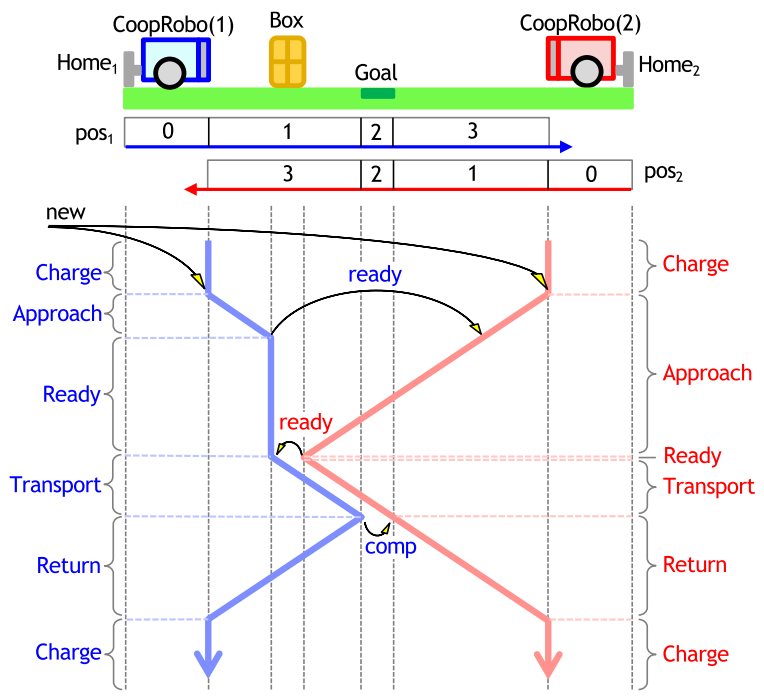

Fig. 3 A basic scenario of cooperative transportation in CoopSys

it is important that sole transportation is forbidden for the safety.

For simplicity, we develop the cooperative robot system on an narrow line (only in one dimension) where the home bases are placed in the ends of the line, the goal is set in the center of the line, and a box appears between the two robots, as shown in Fig. 3. Here, for each robot, the positions are divided to four areas: $\mathbb{0}, 1,2$, and 3 , as shown in $\operatorname{pos}_{i}$ for each CoopRobo(i) in Fig. 3, where the areas 1 and 3 are called the primary area and the non-primary area, respectively. For example, in Fig. 3, the left side of Goal is the primary area for CoopRobo(1) and is the non-primary area for CoopRobo(2).

A basic scenario for cooperatively transporting Box to Goal is also shown in Fig. 3, where each robot has the following five control-modes.

- In the mode Approach, the robot is approaching the box.

- In the mode Ready, the robot has arrived at the box, and is waiting for the other robot.

- In the mode Transport, the robot is cooperatively transporting the box with the other robot.

- In the mode Return, the robot is returning to the home base for charging.

- In the mode Charge, the robot is charging until the battery is full.

In the scenario shown in Fig. 3, at first, a client newly requests both of the two robots to transport a box by the broadcast event new, and then the robots begin approaching the box. When one of the two robots arrives at the box, it notifies the other one to have been ready to cooperatively transport the box by the event ready. Then, when both of the robots arrive at the box, they begin cooperatively transporting the box to the goal, where if the box is placed in the left hand side (i.e. in the primary area for CoopRobo(1)) such as in Fig. 3, the left robot CoopRobo(1) becomes the primary 


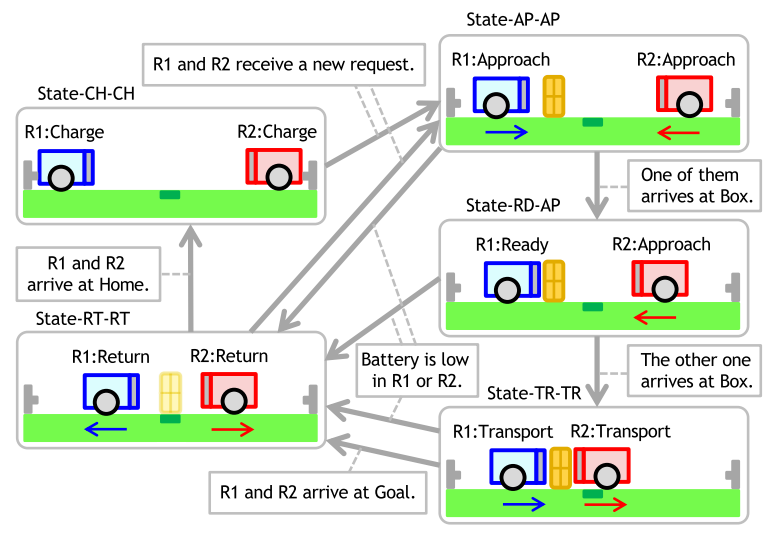

Fig. 4 A part of state-transitions in CoopSys

robot, otherwise the right robot becomes the primary robot. When they arrives at the goal, the primary robot notifies the other one to the completion of the transportation by the event comp. The robots communicate with each other only when they change their mode, and therefore the communication traffic between the two robots is very low.

Figure 4 shows a part of state-transitions in the systemlevel caused by mode-changes of the two cooperative transport robots. Each state in Fig. 4 is explained as follows.

- In State- $\mathrm{CH}-\mathrm{CH}$, both of the robots in the mode Charge are charging at their home bases. When the robots receive a new request (i.e. a new box appears between them), they begin approaching the box, and the state is changed to State-AP-AP.

- In State-AP-AP, both of the robots in the mode Approach are approaching the box. When a robot arrives at the box, the mode of the robot is changed to the mode Ready, and the state is changed to State-RD-AP. If the battery level, which is the remaining battery capacity, of one of the robots is lower than a given threshold Low, the modes of both robots are changed to the mode Return, and this state is changed to State-RT-RT.

- In State-RD-AP, a robot in the mode Ready is waiting for the other robot. When the other robot also arrives at the box, the two robots begin transporting the box, and the state is changed to State-TR-TR. Similarly to the case of State-AP-AP, the state-transition by low battery is also possible.

- In State-TR-TR, the two robots in the mode Transport are cooperatively transporting the box to the goal (the center of the line). When the transportation is completed, the robots begin returning to their home bases, and the state is changed to State-RT-RT. If the battery level of one of the robots is lower than Low, the transportation is suspended until that the batteries of both robots are full.

- In State-RT-RT, the two robots in the mode Return are returning to their home bases. When each robot arrives at its home base, the mode of the robot is changed to the mode Charge. Then, the state is changed to

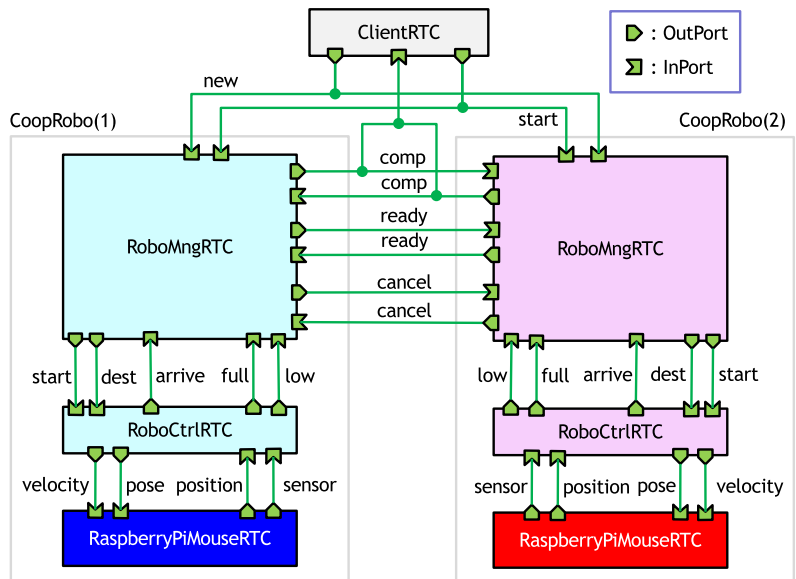

Fig. 5 The structure (RTCs and connections) of CoopSys

Table 1 The events and the meanings in CoopSys

\begin{tabular}{|c|c|}
\hline Event & Meaning \\
\hline $\begin{array}{l}\text { start } \\
\text { new } \\
\text { comp }\end{array}$ & $\begin{array}{l}\text { start CoopSys } \\
\text { make a new request for transportation } \\
\text { notify that the transportation has completed }\end{array}$ \\
\hline $\begin{array}{l}\text { ready } \\
\text { cancel }\end{array}$ & $\begin{array}{l}\text { notify to be ready to transport the box } \\
\text { notify low battery (cancel the ready notification) }\end{array}$ \\
\hline $\begin{array}{l}\text { dest.v } \\
\text { arrive.v } \\
\text { low } \\
\text { full }\end{array}$ & $\begin{array}{l}\text { instruct the destination } v \in\{\text { Home, Goal, Box }\} \\
\text { notify the arrival at the destination } v \\
\text { notify lower battery than the threshold Low } \\
\text { notify full battery }\end{array}$ \\
\hline $\begin{array}{l}\text { velocity.v } \\
\text { pose.v } \\
\text { position.v } \\
\text { sensor.v }\end{array}$ & $\begin{array}{l}\text { set the moving-velocity to } v \\
\text { set the position coordinate value to } v \\
\text { get the current position coordinate value } v \\
\text { get the value } v \text { of the front distance sensor }\end{array}$ \\
\hline
\end{tabular}

State- $\mathrm{CH}-\mathrm{CH}$. To be exact, there can be a state where a robot is on the home base and the other robot is still returning, but it is omitted in Fig. 4. If the robots receive a new request before the arrivals and the batteries of the robots are higher than Low, then the state is changed to State-AP-AP.

In Fig. 4, for briefly explaining the main transitions, we omit some possible states (e.g. State-AP-CH, where a robot is approaching a box and the other robot is charging). The full detail is explained in Sect. 2.3.

\subsection{The Structure of CoopSys}

In this subsection, we design the structure of the cooperative robot system CoopSys on the assumption that it is implemented on RTM (RT-Middleware), which is a software platform for autonomous RTCs (RT-Components), thus CoopSys is a system concurrently composed of RTCs.

The structure of CoopSys is shown in Fig. 5, where each box represents an RTC and each connection represents an event passing between RTCs. The events used in CoopSys are explained in Table 1, where an event can include values $v$ passed with the event, such as dest.v. The system CoopSys consists of two mobile robots CoopRobo(1),(2), and a client Client. The client makes a 


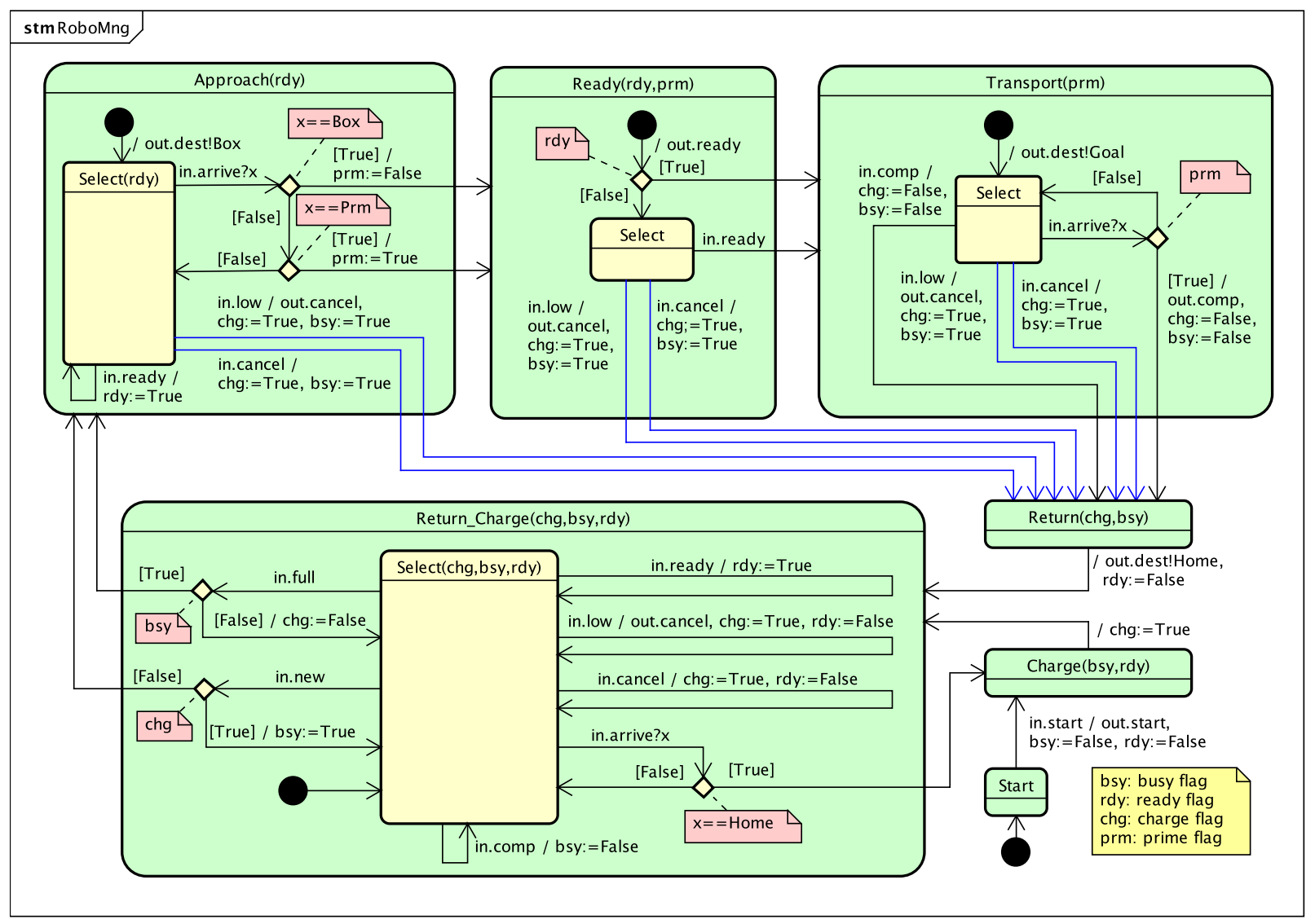

Fig. 6 The finite state machine RoboMng of the RTC RoboMngRTC

request for cooperative transportation by the event new and thereafter receives the completion of the transportation by the event comp. The event start is sent for starting the system. It is noted that the client does not play an important role and it has no interaction with the two robots between the two events, new and comp.

The two mobile robots directly communicate with each other for the cooperative transportation. They are the same robot CoopRobo( $i$ ) except the ID-number $i$, and each robot consists of three RTCs as follows.

- RoboMngRTC is the RTC for managing the controlmode of the robot. For each control-mode, it decides the destination (Home, Goal, or Box), instructs the control component RoboCtrlRTC to go to the destination, and communicates with the other robot for cooperative transportation. This RTC is abstract and is independent of specific robot hardware.

- RoboCtrlRTC is the RTC for controlling the robot hardware RaspberryPiMouseRTC. According to instructions from RoboMngRTC, it decides the moving direction (forward or backward), and instructs the robot hardware to move in the direction. Concurrently, it monitors the position and the sensor values from the robot hardware, and notifies the arrival at the destination by the event arrive. $v$ or the low battery by the event low. The value $v$ of the event arrive.v is one of \{Home, Goal, Box, Prm\}, where Prm (resp. Box) means that the robot has arrived at the box in the primary area (resp. in the non-primary area).

- RaspberryPiMouseRTC is the RTC for Raspberry-Pi Mouse [6], which is a wheel-type robot equipped with a small single-board computer Raspberry Pi. The RTC software for Raspberry-Pi Mouse can be installed from the GitHub [7].

\subsection{The FSMs in CoopSys}

The control logic of each RTC in Fig. 5 is expressed by a finite state machine (FSM). In this subsection, the FSM of each RTC is given and explained.

\subsubsection{The FSM of the RTC RoboMngRTC}

The FSM, named RoboMng, of the RTC RoboMngRTC is shown in Fig. 6 and the flags used in the FSM are explained in Table 2. This FSM has the two-level hierarchy. The five top-level states in Fig. 6, Approach, Ready, Transport, Return, and Charge, correspond to the five control-modes in CoopRobo $(i)$, and the top-level state Return_Charge is the common part of Return and Charge because the responses to events in the two modes are similar. These toplevel states have substates inside them. The top-level initial state is Start and the initial substate inside each state is designated by the filled circle $\bullet$. 
Table 2 The flags used in Fig. 6

\begin{tabular}{|l|ll|}
\hline Flag & Meaning & \\
\hline \hline$b s y$ & busy flag & (a request has been made) \\
$r d y$ & ready flag & (the other robot is ready to transport) \\
chg & charge flag & (full charge is necessary) \\
prm & primary flag & (it manages cooperation) \\
\hline
\end{tabular}

In FSMs, each transition of the form

$$
s \stackrel{\text { in.e? } x / a c t}{\longrightarrow} s^{\prime}
$$

represents that if the input-event $e$ is received in the state $s$ then the variable $x$ is bound to the value received by the event and the action act is performed, and thereafter the state is changed to $s^{\prime}$, where the action act consists of outputevents and/or updates of local variables. The output-event of the form out.e! $v$ represents to send the event $e$ with the value $v$. In this paper, we design FSMs such that every input-event can be always received in every state, where transitions which do not change either states or variables are omitted. For example, the transition by the input-event arrive? $x$ whose source and target are the same substate Select in Ready is possible, but it is omitted in Fig. 6.

In Fig. 6, the transitions from the initial substates (filled circle) have no input-event (e.g. /out.ready) because it is immediately performed. Each diamond $\diamond$ represents a conditional substate, and the transition with [True] (resp. [False]) from the substate is selected if the note (e.g. $x==$ Box) attached to the substate is true (resp. false).

The each state in Fig. 6 is explained as follows.

- The state Start is the top-level initial state. It waits for receiving the event start from the client, and thereafter forwards it to the RTC RoboCtrlRTC and set the two flags, $b s y$ and $r d y$, to False. Then, the state is changed to the state Charge.

- In the state Approach, at first, the event dest with the value Box is sent. It means to set the destination to the box. Thereafter, at the substate Select, the next action is selected in response to the input-event in.e as follows.

- If $e$ is arrive. $v$, then the next action depends on the received value $v$. If $v$ is Box, then the primary flag prm is set to False, and if $v$ is Prm, then prm is set to True. Thereafter, the state is changed to the state Ready.

- If $e$ is ready, then the flag $r d y$ is set to True and the substate Select is not changed.

- If $e$ is cancel or low, then the two flags, chg and $b s y$, are set to True in order to restart approaching after full-charge, and then the state is changed to the state Return. In addition, if $e$ is low then the event cancel is sent for notifying the low battery to the other robot.

- In the state Ready, at first, the event ready is sent for notifying the arrival at Box and if the ready flag $r d y$ is True (i.e. the other robot has been ready) then the state is changed to the state Transport, otherwise it is changed to the substate Select. The next action depends on the input-event in.e as follows.

- If $e$ is ready, then the state is changed to the state Transport because it means that both of the two robots have been ready to transport.

- If $e$ is cancel or low, the next action is the same as the one in the case of the state Approach.

- In the state Transport, at first, the event dest with the value Goal is sent for setting the destination to the goal. Thereafter, at the substate Select, the next action is selected in response to the input-event in.e as follows.

- If $e$ is arrive. $v$ and the prime flag prm is True then the event comp is sent, the two flags, chg and $b s y$, are set to False, and then the state is changed to the state Return. This transition means that the primary robot notifies the completion of the cooperative transportation to the non-primary robot when they arrive at the goal.

- If $e$ is comp, then the two flags, chg and bsy, are set to False, and then the state is changed to the state Return. This transition means that the nonprimary robot receives the completion from the primary robot.

- If $e$ is cancel or low, the next action is the same as the one in the case of the state Approach.

- In the state Return, the event dest with the value Home is sent for setting the destination to the home base. Thereafter, the flag $r d y$ is set to False, and the state is changed to the state Return_Charge, which is the common part with the state Charge.

- In the state Charge, the flag chg is set to True, and the state is changed to the state Return_Charge.

- In the state Return_Charge, at the substate Select, the next action is selected in response to the input-event in.e as follows.

- If $e$ is arrive. $v$ and the received value $v$ is Home, then the state is changed to the state Charge. It means that the control-mode is changed from Return to Charge.

- If $e$ is new, then the next action depends on the charge flag chg. If chg is False then the state is changed to the state Approach, otherwise the busy flag bsy is set to True. It is important to note that the robots can start approaching the new box while they are returning to their home base because the charge flag chg is set to False just after the completion of cooperative transportation.

- If $e$ is full, then the next action depends on the busy flag bsy. If $b s y$ is True then the state is changed to the state Approach, otherwise the charge flag chg is set to False. It is important to note that each robot cannot start approaching while the flag chg is True. 


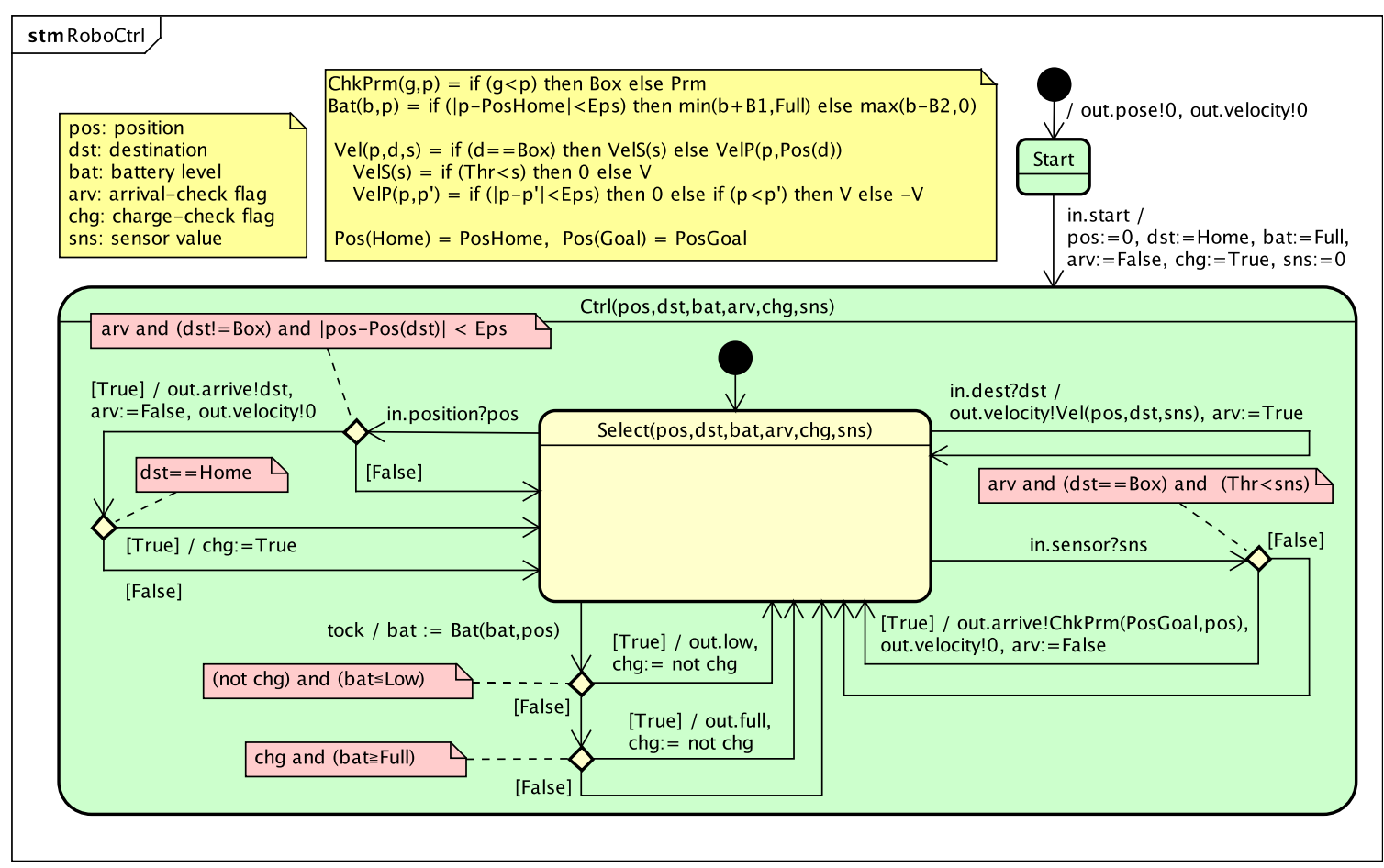

Fig. 7 The finite state machine RoboCtrl of RoboCtrlRTC

- If $e$ is ready, then the ready flag $r d y$ is set to True.

- If $e$ is cancel or low, then the two flags, chg and $r d y$, are set to True and False, respectively. In addition, if $e$ is low then the event cancel is sent.

- If $e$ is comp, then the busy flag rdy is set to False. There is a possibility that the non-primary robot receives the event comp in the state Return_Charge when the two outputevents, out.cancel by the non-primary robot and out.comp by the primary robot, are sent in the state Transport at the almost same time.

\subsubsection{The FSM of the RTC RoboCtrlRTC}

The FSM, named RoboCtrl, of the RTC RoboCtrlRTC is shown in Fig. 7 and the variables used in the FSM are explained in Table 3. The parameters (e.g. V and Thr) used in Fig. 7 are explained in Sect. 3.2 with values for verification as shown in Table 5. RoboCtrlRTC initially resets the position and the velocity of the robot by sending the events pose and velocity, and then waits for receiving the event start from RoboMngRTC. After receiving start, at the substate Select, the next action is selected in response to the input-event in.e as follows.

- If $e$ is dest.v, then the destination variable $d s t$ is bound to the received value $v$, and thereafter, the flag arv is set to True for checking the arrivals at the destination $d s t$ and the event velocity with the velocity $\operatorname{Vel}($ pos, $d s t$, sns), which is defined in the note in Fig. 7, is sent for moving to the destination $d s t$.
Table 3 The variables used in Fig. 7

\begin{tabular}{|l|l|}
\hline Variable & Meaning \\
\hline \hline pos & position of the robot \\
$d s t$ & destination of the robot \\
$b a t$ & battery level (remaining battery capacity) \\
$a v$ & arrival check flag \\
$c h g$ & battery check flag \\
sns & front LED reflective distance sensor value \\
\hline
\end{tabular}

- If $e$ is position.v, then the current position variable pos is updated to the received value $v$ and if the flag arv is True and the destination is not Box (i.e. Home or Goal) and the current position pos is almost equal to the position of the destination $\operatorname{Pos}(d s t)$, then the event arrive with the destination is sent, the event velocity.0 is sent for stopping the robot, and the flag arv is set to False. In addition, if the destination is Home, the charge-check flag chg is set to True for checking the full charge.

- If $e$ is sensor.v, then the sensor variable sns is bound to the received value $v$. Furthermore, if the flag arv is True and the destination is Box and sns is greater than the (given) threshold Thr, then the event arrive.v is sent, where the value $v$ is Prm if the position pos is in the primary area, otherwise $v$ is Box (see the definition of ChkPrm in Fig. 7).

- If $e$ is tock, then the battery level bat is updated by the function $\operatorname{Bat}(b, p)$, which simulates charge and discharge as defined in Fig. 7. Here, tock is the special event that periodically occurs at a control frequency, for example, $50 \mathrm{~Hz}$ in the implementation of this case 


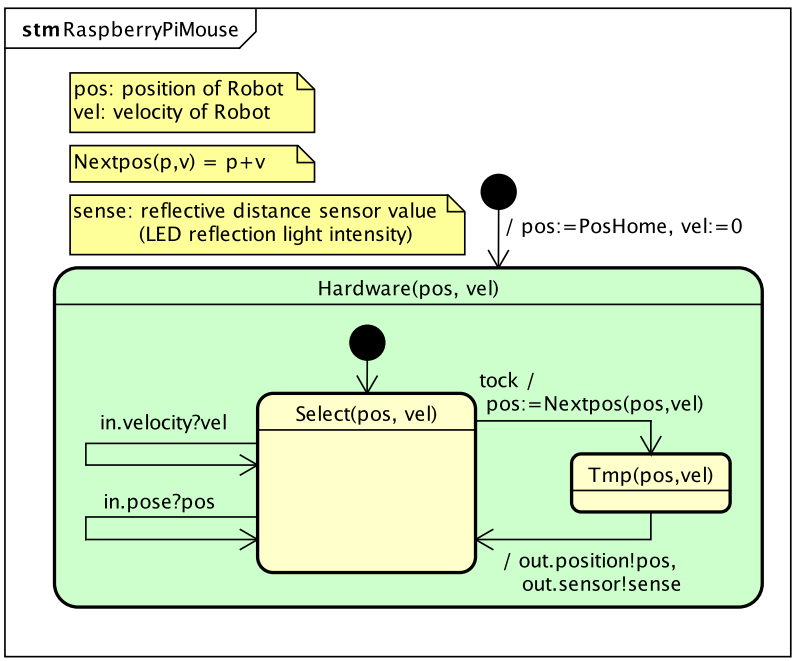

Fig. 8 The finite state machine of the RTC RaspberryPiMouseRTC

study. Thus, RoboCtrlRTC periodically updates the battery level. After each update,

- if the flag chg is False, which means to check the low battery, and the battery level is lower than the threshold Low, then the event low is sent and chg is inverted,

- if the flag chg is True, which means to check the full battery, and the battery level is full, then the event full is sent and chg is inverted,

where the flag chg is used in order to avoid sending the unnecessary extra events low or full.

In this case study, for simplicity, we simulate charge and discharge in RoboCtrlRTC instead of receiving the real battery level from the robot hardware Raspberry-Pi Mouse. In the future, the periodical update of the battery level in RoboCtrlRTC will be replaced with an input-event for receiving the real battery level from the robot hardware.

\subsubsection{The FSM of the RTC RaspberryPiMouseRTC}

The RTC for Raspberry-Pi Mouse has been already given as RaspberryPiMouseRTC in GitHub [7]. Therefore, it is not necessary to design a RTC for Raspberry-Pi Mouse, but the FSM of the RaspberryPiMouseRTC is necessary for verification and simulation.

The FSM, named RaspberryPiMouse, is shown in Fig. 8. Initially, the position variable pos is set to the position of the home base and the velocity variable vel is reset to 0 . Then, at the substate Select in the FSM, the velocity variable vel is bound to the value $v$ received through the event velocity. $v$ and the position variable pos is bound to the value $v$ received through the event pose.v. Furthermore, whenever the periodical event tock occurs, the current position pos is updated by the function Nextpos, and thereafter, the event position with the current position pos and the event sensor with the front LED reflective distance

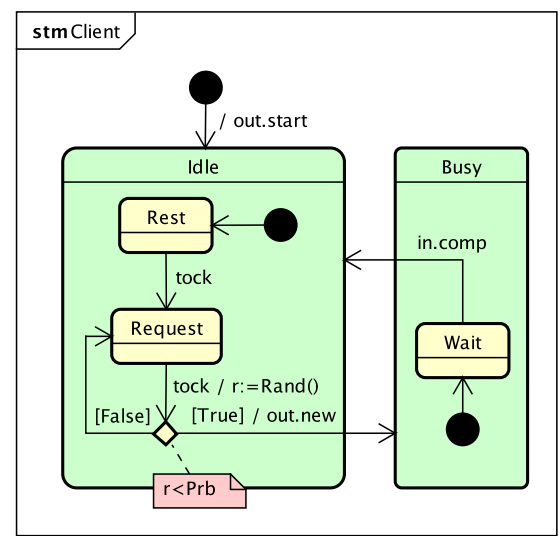

Fig.9 The finite state machine Client of the RTC ClientRTC

sensor value sense are sent. Here, the sensor value sense is the reflection light intensity and it increases as the robot approaches an object.

\subsubsection{The FSM of the RTC ClientRTC}

The FSM, named Client, of the RTC ClientRTC is shown in Fig. 9. The FSM Client initially sends the event start for initializing the system. Then, in the state Idle, after receiving the event tock to wait for one control cycle, at the substate Request, the event new is sent with some delay, and thereafter, the state is changed to the Busy. In the state Busy, Client waits for the event comp at the substate Wait, and then the state returns to the Idle after receiving comp.

It means that a new request cannot be made while the robots are busy in the substate Wait. Note that the robots send the completion event comp just after completion of the cooperative transportation before returning to their home bases. Thus, a new request can be made during the return.

\section{Formalization of CoopSys}

In this section, we give the formal description of the cooperative robot system CoopSys introduced in Sect. 2. At first, in Sect. 3.1, we introduce CSP (Communicating Sequential Processes) [2]-[4], which is one of well known formal description languages for expressing concurrent behaviors. Next, in Sect. 3.2, we formalize the FSMs (e.g. RoboMng), given in Sect. 2.3, in CSP, and then in Sect. 3.3, we formalize CoopSys in CSP by the concurrent composition of the RTCs.

\subsection{Formal Description in CSP}

In CSP, processes, called CSP-processes, are expressed by composition of events, and process-names are used for expressing iterative processes. In this paper, we assume that the set of events Events, ranged over by $a, \ldots$, and the set of process names PNames, ranged over by $N, \ldots$, are given.

The set of CSP-processes Proc, ranged over by 
Table 4 Syntax of CSP-processes ( $P$ and $Q$ are already in Proc)

\begin{tabular}{|l|ll|}
\hline CSP-Process & Meaning & \\
\hline \hline SKIP & successful termination & \\
STOP & deadlock & \\
$a \rightarrow P$ & action prefix & $(a \in$ Events $)$ \\
$P \square Q$ & external choice & \\
$P \sqcap Q$ & internal choice & \\
$P ; Q$ & sequential composition & \\
if $b$ then $P$ else $Q$ & conditional branch & $(b \in$ Bool $)$ \\
$b \& P$ & guard & $(b \in$ Bool $)$ \\
$P[|A|] Q$ & generalized parallel & $(A \subseteq$ Events $)$ \\
$P\|\| Q$ & interleaving & \\
$P \backslash A$ & hiding & $(A \subseteq$ Events $)$ \\
$P \llbracket \mid a \leftarrow a^{\prime} \rrbracket$ & renaming & $\left(a, a^{\prime} \in\right.$ Events $)$ \\
$N$ & process-name & $(N \in$ PNames $)$ \\
\hline
\end{tabular}

$P, Q, \ldots$, is the smallest set including the expressions in Table 4. The operators of CSP are divided to two kinds: $d y$ namic operators for expressing behaviors and static operators for expressing structures. Since the semantics of CSPprocesses is formally given in CSP-books [2]-[4], the meaning of each operator is briefly explained here.

The dynamic operators are used for expressing behaviors as follows. The special process SKIP means the successful termination and STOP means the unexpected termination such as deadlock. The prefix process $a \rightarrow P$ can perform the event $a$ and thereafter behaves like $P$. The branches $P \square Q$ and $P \sqcap Q$ represent choices between $P$ and $Q$, where the choice of $P \square Q$ is externally made by an event of either $P$ or $Q$, while the choice of $P \sqcap Q$ is internally made. The process $P$; $Q$ firstly behaves like $P$, then behaves like $Q$ after the successful termination of $P$. The conditional branch if $b$ then $P$ else $Q$ behaves like $P$ if $b$ is true, otherwise behaves like $Q$. The guarded process $b \& P$ behaves like $P$ if $b$ is true, otherwise stops.

The static operators are used for expressing structures as follows. The parallel process $P[|A|] Q$ represents a concurrent composition of $P$ and $Q$, where they synchronously perform the events included in the set $A$ and independently perform the events not included in $A . P \| Q$ is a syntactic sugar of $P[|\emptyset|] Q$, thus $P$ and $Q$ independently performs all the events. The process $P \backslash A$ behaves like $P$ except that the events included in $A$ are hidden. The process $P \llbracket a \leftarrow a^{\prime} \rrbracket$ behaves like $P$ except that the event $a$ is renamed to $a^{\prime}$.

A process name $N \in$ PNames is a process whose meaning is given by a defining equation of the form $N=P$, where $P \in$ Proc. For example, the following process name Mutex iteratively performs the events lock and unlock alternately.

$$
\text { Mutex }=\text { lock } \rightarrow \text { unlock } \rightarrow \text { Mutex }
$$

In CSP, the dot '.' is used as a constructor for expressing parameterized events (e.g. ev. $x$ ). For example, the event ev with the parameters $1, \ldots, 6$ is described by ev. $1, \ldots$, ev.6. For expressing sets of such parameterized events, the syntactic sugar $\{|\cdot|\}$ is often used as follows:

$$
\{|\mathrm{ev}|\}=\{\mathrm{ev} . x \mid x \in\{1, \ldots, 6\}\}=\{\mathrm{ev} .1, \ldots, \mathrm{ev} .6\} .
$$

In CSP, some syntactic sugars of operators are also de- fined for convenience. The following replicated operators are used in this paper:

$$
\begin{aligned}
& \square x: S \bullet P(x): \text { replicated external choice, } \\
& \sqcap x: S \bullet P(x): \text { replicated internal choice, and } \\
& \| x: S \bullet P(x) \quad: \text { replicated interleaving, }
\end{aligned}
$$

where $S$ is a finite index set. For example, in the following process Dice, one of the six events ev.1, ., ev.6 is iteratively non-deterministically chosen:

$$
\begin{aligned}
\text { Dice } & =\sqcap x:\{1, \ldots, 6\} \bullet(\text { ev. } x \rightarrow \text { Dice }) \\
& =(\text { ev. } 1 \rightarrow \text { Dice }) \sqcap \cdots \sqcap(\text { ev.6 } \rightarrow \text { Dice })
\end{aligned}
$$

CSP-processes do not only synchronize by events as explained above, but also can pass values through synchronous channels, as expressed by the following syntactic sugar:

$$
\begin{aligned}
\operatorname{ch} ! v \rightarrow P & =\text { ch.v } \rightarrow P, \\
\operatorname{ch} ? x \rightarrow P(x) & =\square x: \operatorname{val}(c h) \bullet(\text { ch. } x \rightarrow P(x)),
\end{aligned}
$$

where val $(c h)=\{v \mid$ ch. $v \in$ Events $\}$ is the set of values passed through the channel $c h$. The event $c h ! v$ means sending the value $v$ to the channel $c h$ and the event $c h ? x$ means receiving a value from the channel $c h$ and binding $x$ to the value.

In CSP, the basic communication is synchronous, thus a sending event and receiving events must synchronize. However, asynchronous communications can be also expressed by inserting buffers as explained in Sect. 3.3.

\subsection{Formalization of FSMs in RT-Components}

In this subsection, we give the CSP-processes of the FSMs explained in Sect. 2.3.

\subsubsection{The CSP-Process of RoboMng}

Figure 10 shows the CSP-process corresponding to the FSM RoboMng in Fig. 6, where the process-definitions for $\operatorname{Ready}(r d y, p r m)$ and Transport (prm) are omitted because they can be described similarly to $\operatorname{Approach}(r d y)$. In Fig. 10, the let-expression (let ... within $\cdots$ ) is used for locally defining processes without conflicts of processnames. For example, the let-expression of Approach $(r d y)$ (lines 3-17) shows that Approach $(r d y)$ (line 3) is equal to Init (line 17) which is defined in the 4th line.

The CSP-process Approach $(r d y)$ in Fig. 10 expresses the behavior inside the top-level state $\operatorname{Approach}(r d y)$ in Fig. 6 except that the event obs.APPROACH.ID is inserted at the first of Init. The events obs.* are used for observing states in verification by model checking. For example, obs.APPROACH.ID represents that CoopRobo(ID) starts approaching. The CSP-process Select $(r d y)$ in the 5th line represents to receive one of the events arrive.v, low, cancel, ..., and tock, where the choice is decided by the event sent from the other processes because the external choice $\square$ is used. The CSP-processes for the other behaviors in Fig. 6 are described in a similar way. 


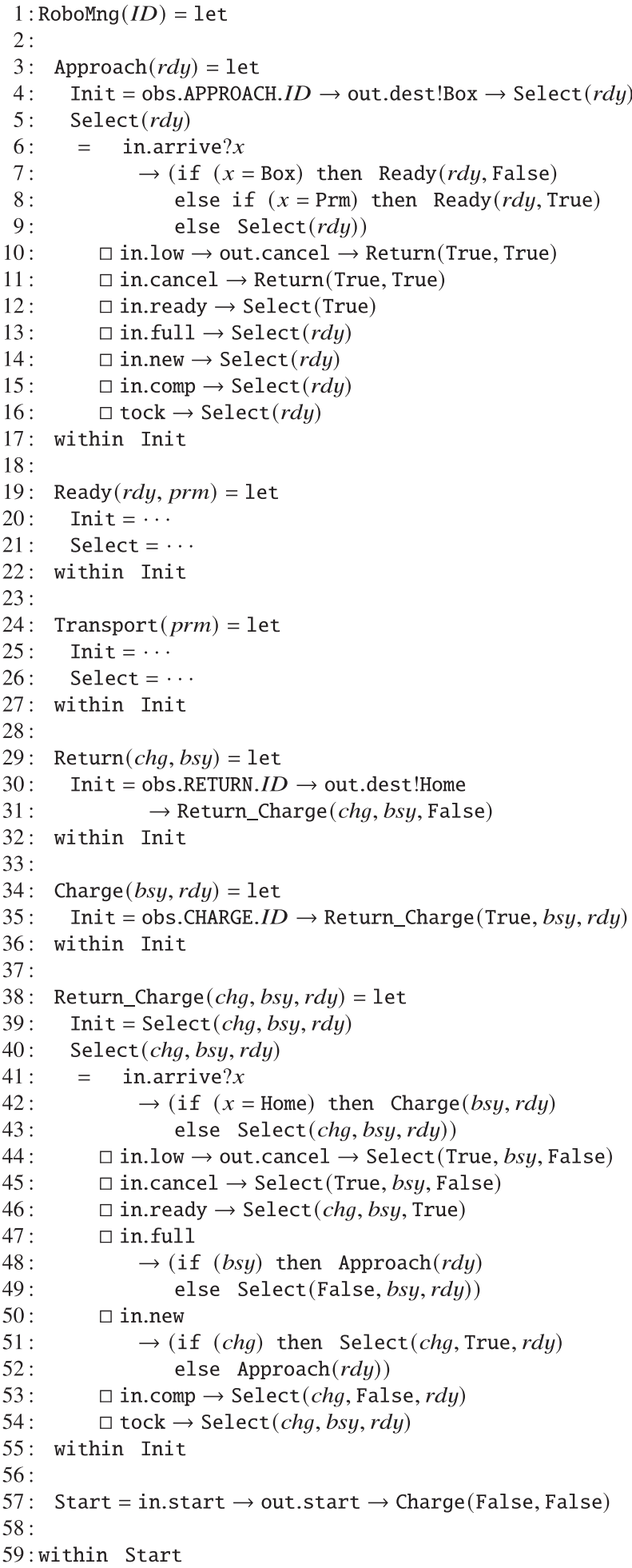

Fig. 10 The CSP-process of the FSM RoboMng

\subsubsection{The CSP-Process of RoboCtrl}

Similarly to RoboMng, the FSM RoboCtrl in Fig. 7 is also formally described in CSP as shown in Fig. 11 except that

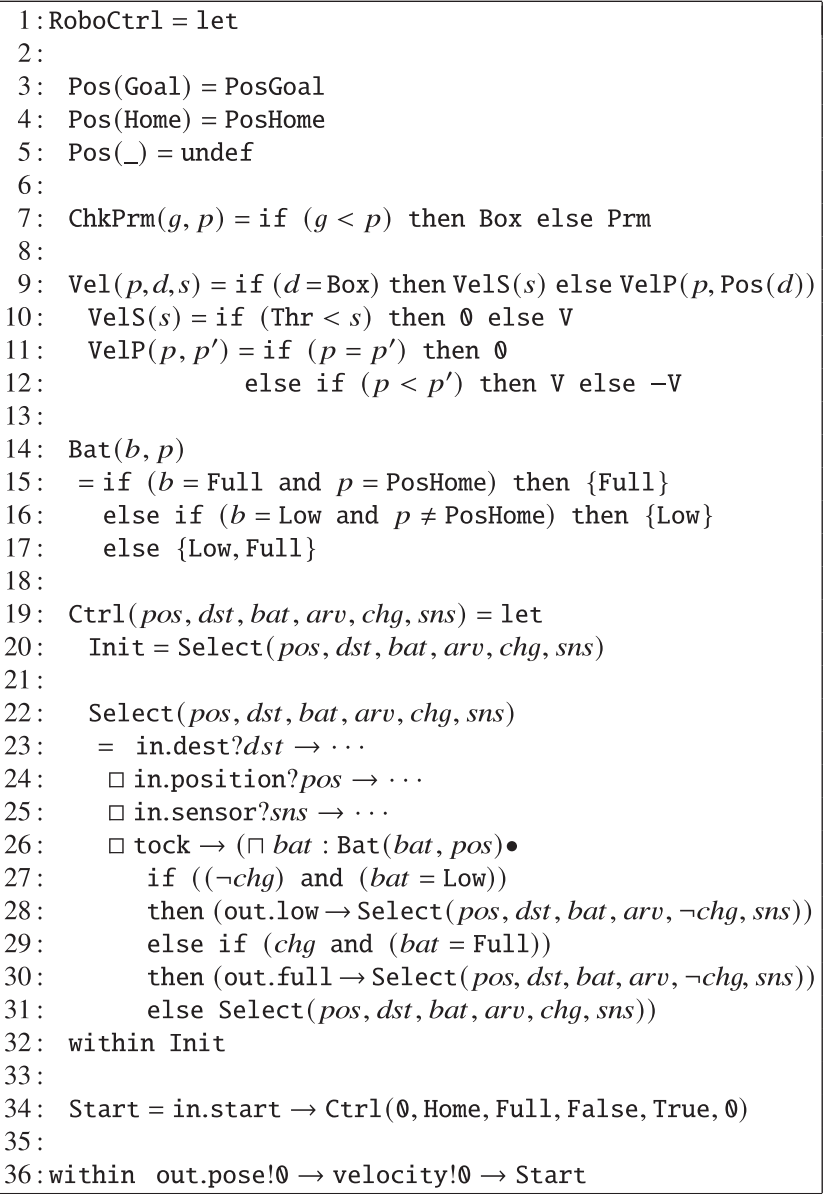

:

Fig. 11 The CSP-process of the FSM RoboCtrl

the continuous battery level bat is abstracted to the set of only two levels $\{$ Low, Full $\}$ for avoiding state-space explosion. In this case study, the integer values 0 and 1 are assigned to Low and Full, respectively, for convenience. In order to cover the wide-range of the continuous battery level by the two levels, the internal choice $\Pi$ is used (line 26). It means that the battery level bat non-deterministically decreases or remains or increases, in accordance with the set of possibilities $\operatorname{Bat}(b, p)$ defined in the 14th line, which depends on the battery level $b$ and the position $p$. For example, the battery level Full non-deterministically changes to Low or Full when the robot is not in the home base.

\subsubsection{The CSP-Process of RaspberryPiMouse}

The FSM RaspberryPiMouse is formally described in CSP as shown in Fig. 12. Similarly to RoboCtrl, the continuous position pos is abstracted to the set of only discrete four positions $\{0,1,2,3\}$ for avoiding state-space explosion. The four positions of the two cooperative robots CoopRobo(1) and CoopRobo(2) are shown as pos 1 and pos 2 in Fig. 3, respectively. Therefore, the four abstract discrete positions correspond to the four areas explained in Sect. 2.1. The internal choice $\Pi$ with the function $\operatorname{Nextpos}(p, v)$ in Fig. 12 (line 16) means that each robot goes to the next position $p+v$ 


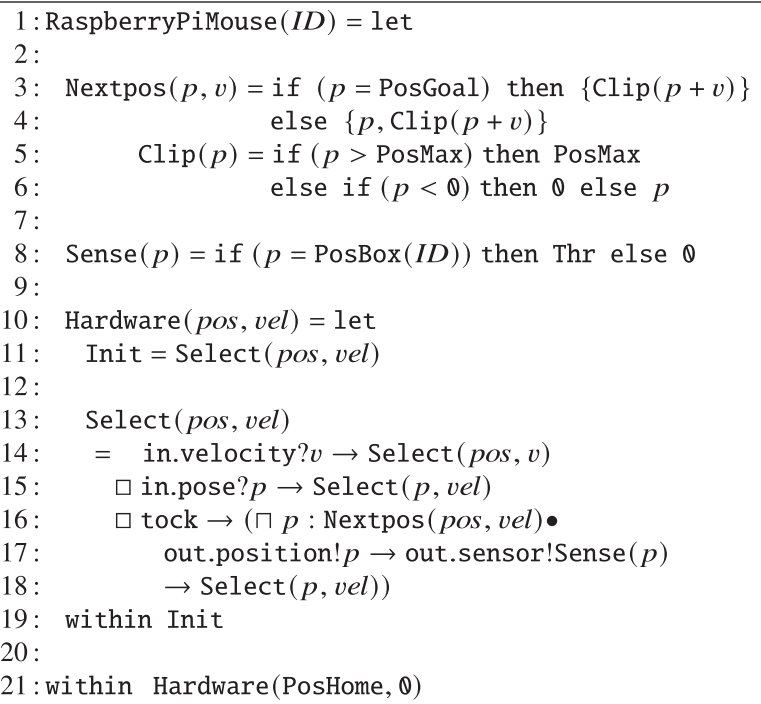

Fig. 12 The CSP-process of the FSM RaspberryPiMouse

Table 5 The parameters in CoopSys

\begin{tabular}{|l|c|l|}
\hline Parameter & Value & Meaning \\
\hline \hline PosMax & 3 & maximum position \\
PosHome & 0 & home position \\
PosGoal & 2 & goal position \\
PosBox(1) & 3 & box position for CoopRobo $(1)$ \\
PosBox $(2)$ & 1 & box position for CoopRobo $(2)$ \\
V & 1 & moving speed \\
Low & 0 & low battery-level \\
Full & 1 & full battery-level \\
Thr & 1 & threshold for sensing boxes \\
\hline
\end{tabular}

or stay in the same position $p$, non-deterministically, whenever the time event tock occurs, where the moving velocity $v$ is either $-1,0$, or 1 (i.e. the parameter $\mathrm{V}$ in Fig. 11 is 1 ). The sensed value Sense $(p)$ is also abstracted to two values $\{0, T h r\}$ (line 8) and the parameter PosBox(ID) represents the position of the box.

The parameters used in Figs. 11 and 12 and their values for verification in this case study are summarized in Table 5.

\subsubsection{The CSP-Process of Client}

The FSM Client is formally and easily described in CSP as shown in Fig. 13. The behavior of the CSP-process corresponds to the FSM except that the probabilistic choice in FSM is replaced by the non-deterministic choice $\square$ (line 6).

\subsection{Formalization of Systems on RTM}

In this subsection, we formalize the the cooperative robot system CoopSys by concurrently connecting the sequential CSP-processes of the FSMs given in Sect. 3.2 in accordance with the communication style of RT-Middleware (RTM). At first, we present the generic (reusable) CSP-process for RTM, and then explain the CSP-process for CoopSys on RTM.

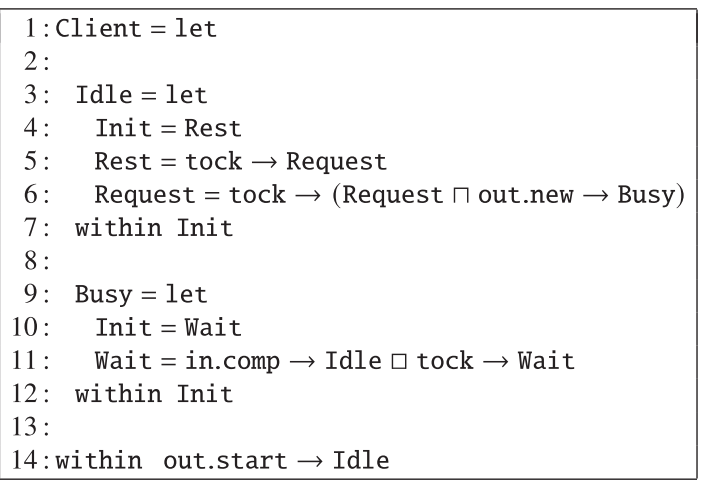

Fig. 13 The CSP-process of the FSM Client

\subsubsection{The CSP-Process of RTM}

RT-Middleware (RTM) connects RT-Components (RTCs) through channels for broadcast communications with ring buffers, where

- the broadcast communication means that an event sent by an RTC can be received by a number of RTCs which request to receive it,

- the ring buffer means that the oldest event in the buffer can be overwritten by a new event if the buffer is full and the overwriting-flag is true (if the flag is false, the overwriting is blocked).

The parallel composition [| $A \mid]$ in CSP connects events (channels) with the same names included in $A$. In order to connect the CSP-processes of FSMs defined in Sect. 3.2, the events are carefully renamed by the operator $\llbracket a \leftarrow a^{\prime} \rrbracket$ and sets $A$ of synchronous events are defined, but it is more difficult and more error-prone than describing sequential CSPprocesses (e.g. Fig. 10) of the FSMs.

Therefore, we give the generic and compact CSP description as shown in Fig. 14, for connecting any RTCs with FSMs in accordance with the communication style of RTM, where the events and the sets in the CSP-process are summarized in Tables 6 and 7, respectively. The first line in Fig. 14

\section{RTM_compo(FSM,link, $c, w)$}

means that the finite state machines $F S M(i){ }_{\left(i \in \mathrm{RTC}_{\text {_id }}\right)}$ are connected by the link-information $\operatorname{link}(i, j)$, which is the set of events passing from $F S M(i)$ to $F S M(j)$, in accordance with the communication style of RTM, where $c$ is the capacity of ring buffers and $w$ is the overwriting-flag.

In Fig. 14, the ring buffer, named $\operatorname{Buff}(i)$, is defined (lines 3-9), where $s$ is the list, whose length is less than or equal to the capacity $c$, for storing events, and if the buffer is full and the overwriting flag $w$ is true (line 8) then the oldest event (i.e. the head of the list $s$ ) is overwritten by the new event $e$ and the event ow. $i$ occurs for notifying that the oldest event has been overwritten in the buffer of the $i$-th RTC. 


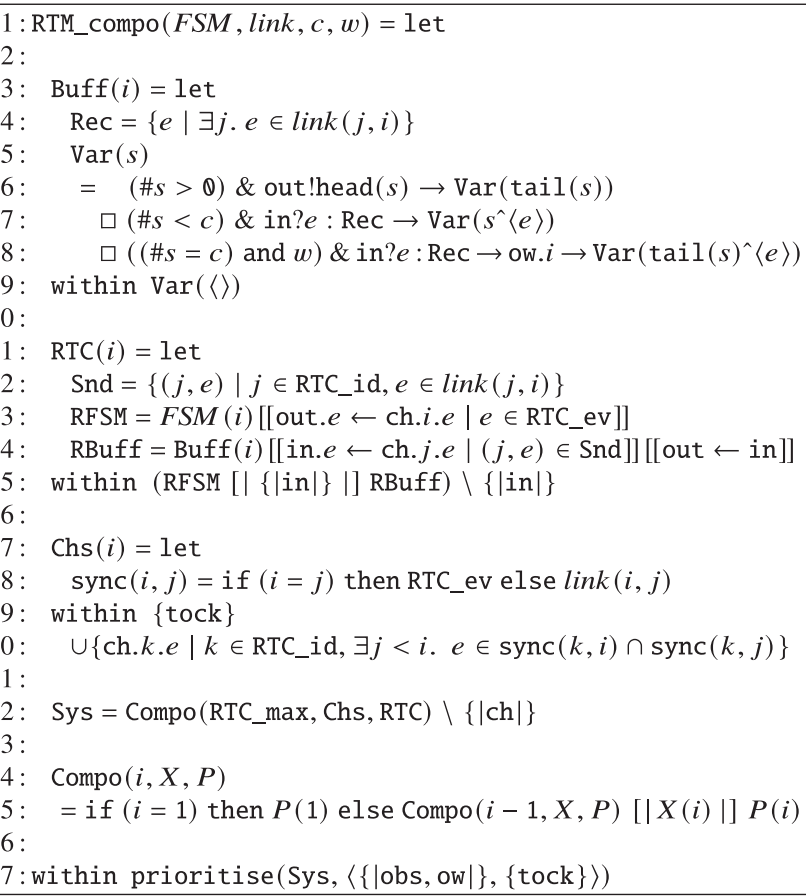

Fig. 14 The CSP-process of the RT-Middleware

Table 6 The CSP-events used in RTM_compo

\begin{tabular}{|l|l|}
\hline Event & Meaning ( $e \in$ RTC_ev, $i \in$ RTC_id, $m \in$ RTC_msg) \\
\hline \hline in. $e$ & receive the event $e$ \\
out. $e$ & send the event $e$ \\
ch.i.e & pass the event $e$ sent by the $i$-th RTC \\
obs. $m$ & observe the message $m$ \\
ow. $i$ & notify an overwriting in the $i$-th buffer \\
tock & represent the passage of one time unit \\
\hline
\end{tabular}

Table 7 The sets used in RTM_compo

\begin{tabular}{|l|l|}
\hline Set & Meaning \\
\hline \hline RTC_id & the set of RTC-IDs (\{1,.., RTC_max $\})$ \\
RTC_ev & the set of event-messages used in FSMs \\
RTC_msg & the set of observable messages for verification \\
\hline
\end{tabular}

In order to define the $i$-th RTC with the $F S M(i)$, each output event out.e from FSM(i) is renamed to ch.i.e (line 13) while each input event in.e to $\operatorname{Buff}(i)$ is renamed to ch.j.e if $e \in \operatorname{link}(j, i)$ (line 14). Then, RTC $(i)$ is the composition of the renamed FSM (RFSM) and the renamed buffer (RBuff), where the output out from the buffer is connected to the input of the FSM and is hidden (line 15).

Finally, for each $i \in$ RTC_id, RTC $(i)$ is recursively connected to the others $\operatorname{RTC}(j)$ such that $j<i$ through the events ch.k.e $\in \mathrm{Chs}(i)$ (line 22) by the function Compo ${ }^{\dagger}$ (lines 2425 ), where Chs $(i)$ is defined (lines 17-20) for synchronizing through the events which either $\operatorname{RTC}(j)$ receives from $\operatorname{RTC}(i)$,

${ }^{\dagger}$ In CSP, the replicated alphabetized parallel composition is often used for the composition of a number of CSP-processes, but it requires that all the shared events must synchronize. In this paper, we define the composition Compo for independently performing the observations obs.

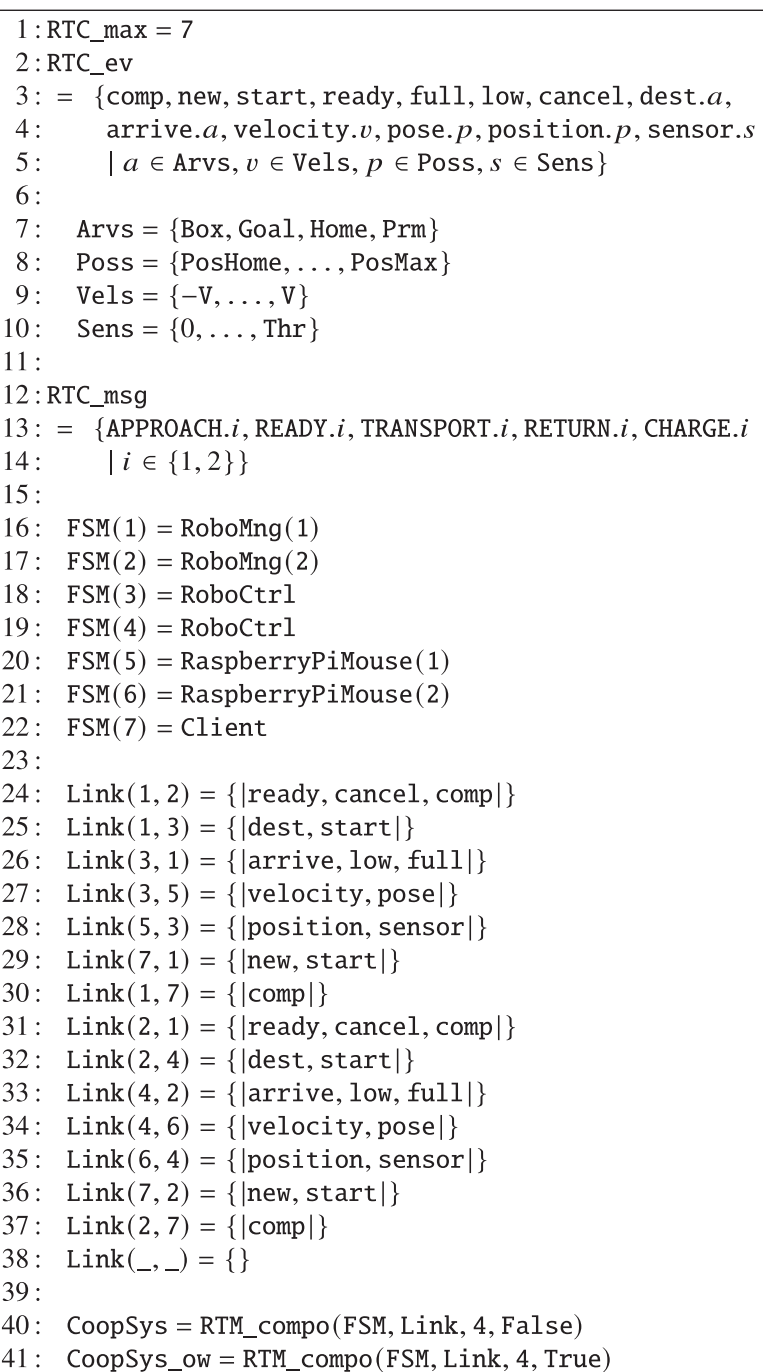

Fig. 15 The CSP-process of CoopSys

or $\operatorname{RTC}(i)$ receives from $\operatorname{RTC}(j)$, or both $\operatorname{RTC}(i)$ and $\operatorname{RTC}(j)$ receive from $\operatorname{RTC}(k)$.

In this paper, we assume that internal communications (hidden events such as ch) and observations obs have priority over time passage tock, similarly to the assumption in Timed-CSP [15]. The priority is defined at the 27th line in Fig. 14.

\subsubsection{The CSP-Process of CoopSys}

The CSP-process of the cooperative robot system CoopSys is defined in Fig. 15. In the upper part (lines 1-14) of Fig. 15, the sets used in RTM_compo (see Table 7) are defined. In the other part, the function FSM(i) (lines 16-22) assigns the CSP-process of the FSM to the $i$-th RTC in Coopsys and the function $\operatorname{Link}(i, j)$ (lines 24-38) defines the set of the events passing from the $i$-th RTC to the $j$-th RTC. The structure made by $\operatorname{FSM}(i)$ and $\operatorname{Link}(i, j)$ corresponds to the one in Fig. 5. For example,

$$
\operatorname{FSM}(1)=\operatorname{RoboMng}(1),
$$




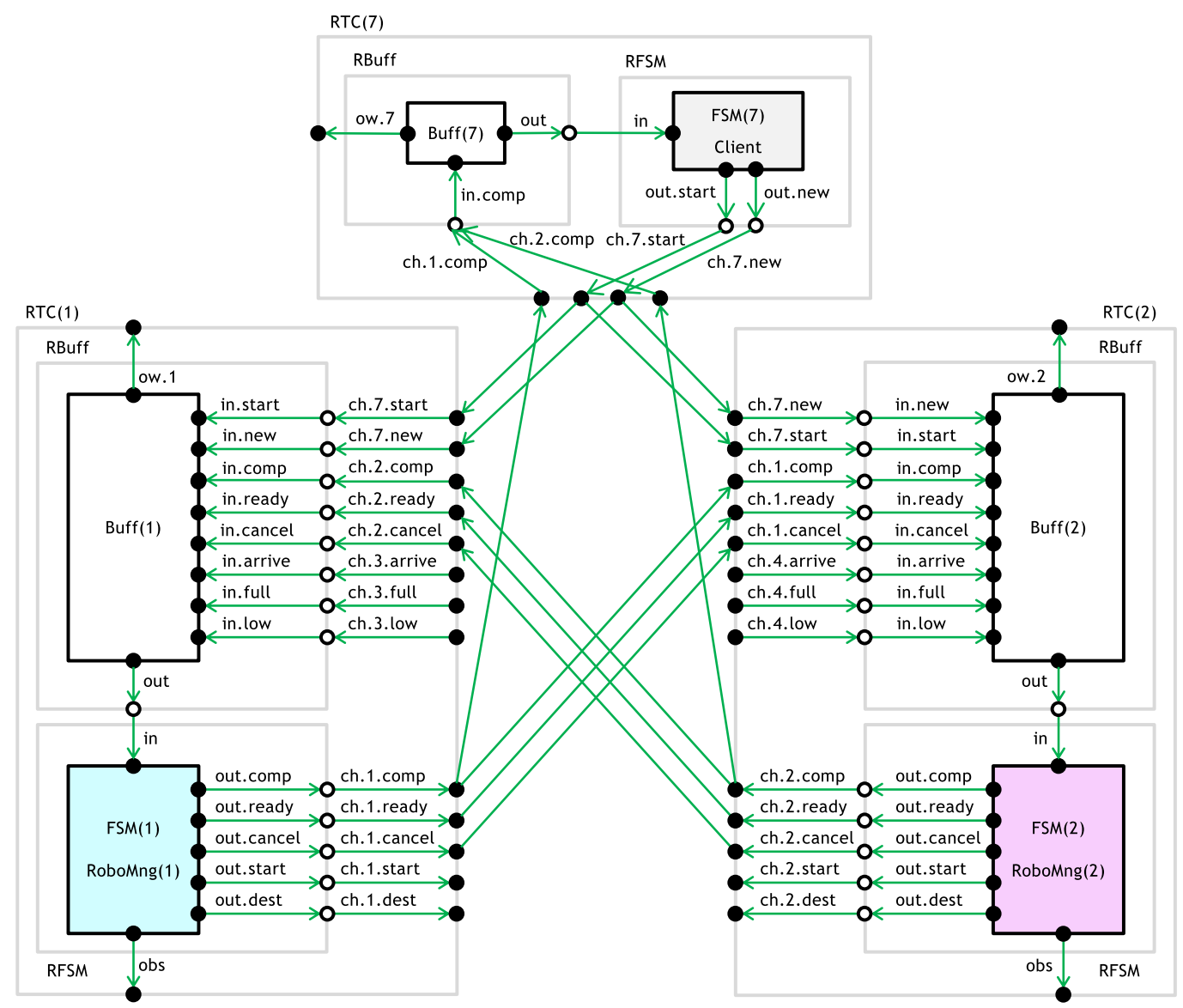

Fig. 16 The composition of RoboMng(1), RoboMng(2), and Client by RTM_compo( $\cdots)$

$$
\begin{aligned}
\operatorname{FSM}(3) & =\text { RoboCtrl }, \text { and } \\
\operatorname{Link}(1,3) & =\{\mid \text { dest, start } \mid\}
\end{aligned}
$$

mean that two connections exist for sending the events dest and start from RoboMngRTC(1) to RoboCtrlRTC. Then, as shown at the 40th line in Fig. 15, the CSP-process of CoopSys is the concurrent composition of FSM $(i)_{\left(i \in R_{T C} \text { id }\right)}$, which are connected by the events in $\operatorname{Link}(i, j)$, where the capacity of every ring-buffer is 4 and overwritingflag is false (i.e. overwriting is blocked), Furthermore, CoopSys_ow is also defined for checking whether buffers are overwritten or not when overwriting is allowed.

In order to help to understand how RTM_compo connects FSMs, we show the connections between RoboMng(1), RoboMng(2), and Client in Fig. 16. It is a part of the structure of the cooperative robot system CoopSys composed by RTM_compo, where each white small circle represents a renaming.

\section{Verification of CoopSys}

In Sects. 2.3 and 3.2, the individual behaviors of the RTCs (ClientRTC, RoboMngRTC, RoboCtrlRTC, and RaspberryPiMouseRTC) in the cooperative robot system CoopSys have been clearly and formally explained by FSMs and CSP-processes. It is, however, difficult to exactly under- stand the whole concurrent behavior of CoopSys because it is often non-deterministic and sometimes causes unexpected states due to interactions between the RTCs.

In this section, we explain how to exhaustively verify cooperative behaviors in CSP. At first, in Sect.4.1, we introduce a refinement relation for the verification. Next, in Sect. 4.2, we define specifications of CoopSys. Then, in Sect. 4.3, we show the verification results of CoopSys, where we explain how design-errors in an FSM are detected and corrected.

\subsection{Trace-Refinement in CSP}

It is difficult to detect errors in cooperative behaviors by testing because such errors are non-deterministic and rarely occur. Such cooperative behaviors can be formally described in CSP as explained in Sect. 3 and can be formally verified in the theory of CSP.

In CSP, various refinement relations between two CSPprocesses are defined. In this subsection, we introduce the simplest refinement relation called traces-refinement. One CSP-process $P$ is said to be a traces-refinement of another CSP-process $Q$, written $Q \sqsubseteq_{\mathrm{T}} P$, if and only if

if $P$ can perform a trace (a sequence of events), then $Q$ can also perform the trace. 


\begin{tabular}{|ll|}
\hline (A1) & SafeSpec $\sqsubseteq_{\mathrm{T}}$ CoopSys \\
(A2) & CoopSys $\backslash$ tock $\} \sqsubseteq_{\mathrm{T}}$ TestCase \\
(A3) & CoopSys $\backslash\{$ obs $\mid\}$ is deadlock free \\
(A4) & CoopSys $\backslash\{$ obs $\mid\}$ is livelock free \\
(A5) & CoopSys $\sqsubseteq_{\mathrm{T}}$ CoopSys_ow
\end{tabular}

Fig. 17 The assertions for CoopSys

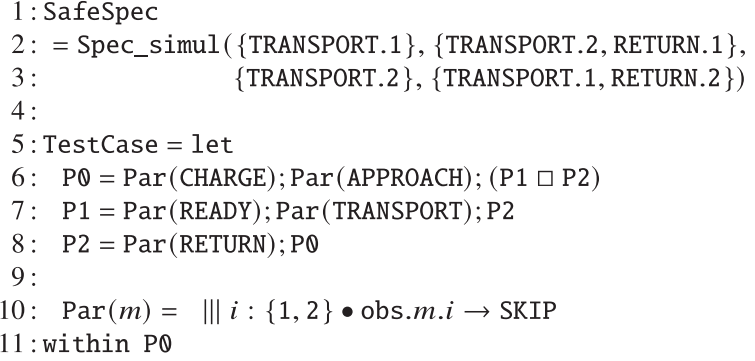

Fig. 18 The specifications for CoopSys

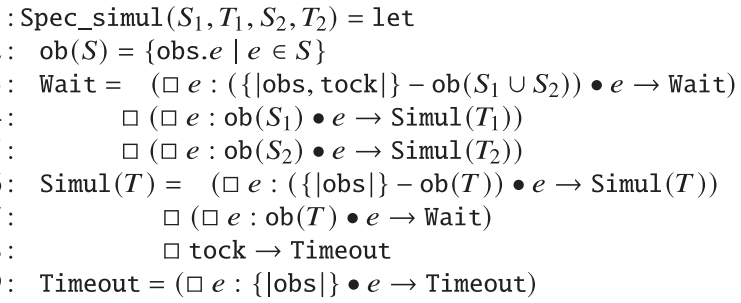

Fig. 19 The reusable description for RTM-Specifications

Therefore, the traces-refinement can guarantee that $P$ never perform traces (usually, critical traces) which $Q$ does not perform. For example, the traces-refinement (A1) in Fig. 17 is used for checking whether the cooperative robot system CoopSys never solely transport boxes (i.e. never perform the critical operation), where CoopSys is the CSP-process defined in Fig. 15 and SafeSpec is a CSP process which is defined in the next Sect. 4.2 and contains only traces for cooperative transportation (not sole transportation). It is an important advantage of the verification based on the tracesrefinement compared with testing because it is difficult for testing to guarantee that a non-deterministic process never perform a trace.

\subsection{The Assertions for CoopSys}

Figure 17 shows the assertions which Coopsys is expected to satisfy. Each assertion is explained below.

As mentioned at the end of the previous Sect. 4.1, the first assertion (A1) in Fig. 17 means that CoopSys never solely transport boxes. The CSP-process of the specification SafeSpec is defined in Fig. 18 (lines 1-3), where Spec_simul $\left(S_{1}, T_{1}, S_{2}, T_{2}\right)$ is a reusable generic specification defined in Fig. 19 and it contains all the traces which satisfies the following condition:

when an event in the set $S_{1}$ (resp. $S_{2}$ ) is performed, an event in the set $T_{1}$ (resp. $T_{2}$ ) must be performed before one control cycle (tock) has passed.

For instance, SafeSpec contains all the traces which satisfies the following condition: for each $i \in\{1,2\}$,

within one control cycle after that CoopRobo(i) has entered the Transport mode, either CoopRobo $(3-i)$ must enter the Transport mode or CoopRobo(i) must enter the Return mode.

It is noted that CoopRobo $(i)$ may cancel the transportation just after entering the Transport mode due to the low battery. Consequently, if the refinement relation (A1) holds, then CoopRobo(1) and CoopRobo(2) necessarily begin transporting a box at almost the same time, where the time difference is less than one tock.

The first assertion (A1) can guarantee the non-existence of the critical traces to sole transportation, but it cannot guarantee the existence of any necessary traces, for example, SafeSpec $\sqsubseteq_{\mathrm{T}}$ STOP holds. Therefore, the existence of typical traces is also checked by the second assertion (A2) in Fig. 17, which means that CoopSys can perform typical traces contained in TestCase defined in Fig. 18 (lines 5-11). In other words, the two robots CoopRobo(1) and CoopRobo(2) can charge the battery, and then approach a box, and then either return home or be ready, and then cooperatively transport the box, and then return home, where time-constraint is ignored by hiding tock for simplicity.

The third assertion (A3) guarantees that time never stops, where all the events obs.* except tock are hidden. CSP-processes carelessly designed often deadlock and they unrealistically stop time. For example, if the capacity of every buffer in CoopSys is 1 , then (A3) does not holds.

The forth assertion (A4) guarantees that infinitely many events are never performed within one control-cycle tock. When all the events except tock are hidden, if internal loops (i.e. livelocks) exist, then it means that any number of events can be performed without tock. Such a design is unrealistic and should be corrected.

The fifth assertion (A5) guarantees that overwriting in buffers never occurs in CoopSys_ow because the event ow.* (see Fig. 14, line 8) is never performed in CoopSys, where CoopSys_ow is the same as CoopSys except that overwriting is allowed in CoopSys_ow. Therefore, in other words, (A5) guarantees that writing in buffers is never blocked in CoopSys.

\subsection{Model Checking by FDR}

The model checker FDR (Failures-Divergences Refinement) is a tool for checking whether refinement relations between CSP-processes written in CSPM (Machine-readable CSP) holds or not, where CSPM is the formal specification language combining the operators of CSP with a functional language. For example, all the CSP-processes of the cooperative robot system CoopSys defined in Sect. 3 and the specifications SafeSpec and TestCase defined in Sect. 4.2 can be completely described in CSPM by trivial syntactical translation to the ASCII-characters (e.g. the symbols $\rightarrow$ and $\square$ in 

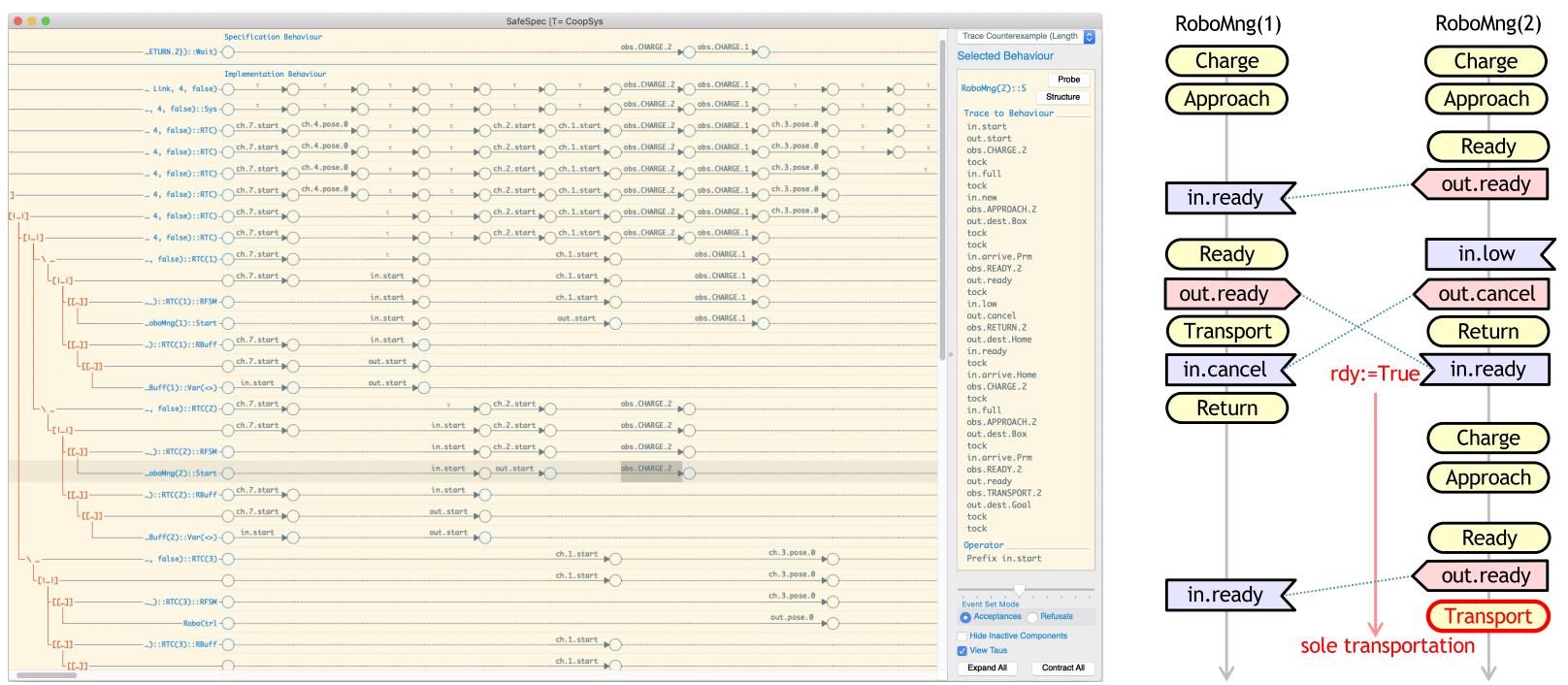

Fig. 21 The debugger for analyzing a trace to sole transportation and the two extracted sub-traces

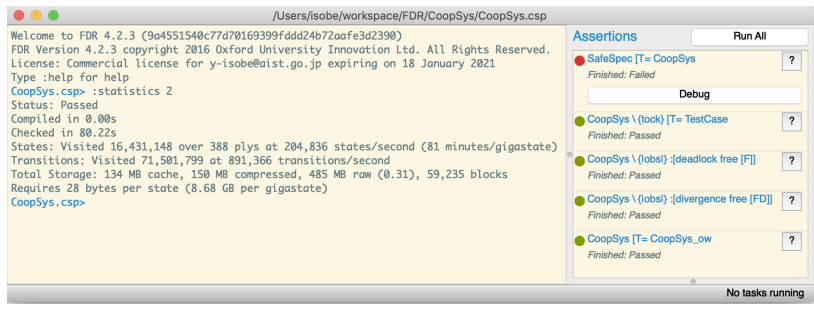

Fig. 20 The results of checking by FDR for the assertions in Fig. 17

CSP are replaced with -> and [] in CSPM, respectively). All the CSPM codes for CoopSys can be downloaded from the web-site [16].

All the assertions in Fig. 17 can be automatically checked by FDR, and the verification results are shown in Fig. 20. The left pane in Fig. 20 shows that FDR (ver.4.2.3) checked all the 16,431,148 states and all the 71,501,799 transitions in CoopSys for the third assertion (A3) and it took about 80 seconds in MacBook-Pro (macOS: High Sierra, CPU: 3.5GHz Core-i7, MEM: 16GB).

The right pane in Fig. 20 shows the result of the check for all the assertions in Fig. 17. Consequently, the assertion (A1) does not hold and the other assertions hold. The result for the assertion (A1) means that sole transportation is possible.

By clicking the button Debug in Fig. 20, a counterexample for the assertion (A1) is displayed in the debugger as shown in the left side of Fig. 21. The counter-example is a critical trace, whose length is 192 , leading to the sole transportation. The critical trace can be analyzed by decomposing it to component-wise partial traces. For example, the partial trace related to RoboMng(2) included in the critical trace is displayed in the right pane of the debugger in Fig. 21.

The two abstract partial traces related to RoboMng(1) and RoboMng(2) extracted from the critical trace are shown in the right side of Fig. 21. The interaction of the two abstract partial traces shows that the sole transportation is caused by the almost simultaneous occurrence of the two output-events out.ready and out.cancel. In this case, CoopRobo(2) solely transports a box because the ready-flag $r d y$ remains True after receiving the event ready.

As explained above, the sole transportation is possible due to design-errors, which are related to event-messages between the transport robots, in the FSM RoboMng of Fig. 6, and it is important to note that the design-errors can be detected before implementation. It is difficult to detect the design-errors by testing because the errors can be observed when the two events ready and cancel simultaneously occur.

The critical trace can be removed by adding the outputevent out.cancel on the two transitions from the substates Select in the two states Ready and Transport to the state Return in the FSM RoboMng in Fig. 6 as follows:

$$
\text { Select } \stackrel{\text { in.cancel/out.cancel, chg:=True, } b s y:=\text { True }}{\longrightarrow} \operatorname{Return}(\ldots)
$$

The correctness for the addition of the output-events can be verified by modifying the CSP-process of RoboMng in Fig. 10, and then by checking all the assertions in Fig. 17 by FDR, again. In fact, all the assertions hold by the modification.

Concurrent FSMs should be carefully modified because careless modifications can easily cause the other faults. For example, if the output-event out.cancel is added only on the transition from the substates Select in Ready (i.e. not in Transport) to the state Return, then the other critical trace to sole transportation is generated by model-checking for the assertion (A1). And if the output-event out.cancel is also added on the transition by in.cancel in Return_Charge, then a livelock is detected by model-checking for the assertion (A4).

It is important to note that the sole transportation found in Fig. 21 cannot be easily avoided by reconfirming the 
ready-state of the other robot by redundant events just prior to starting the transportation. Such redundant events are practically important for the safety and will reduce the possibility of the sole transportation, but they may cause the other (very rare) critical traces. Necessary events for correct design and redundant events for the safety should be distinguished.

\subsection{Scalability of Model Checking}

The model checking approach used in this paper is not scalable to the number of RTCs because the number of reachable states in the system consisting of RTCs exponentially increases with respect to the number of concurrent RTCs in general. For example, it is easy to formally describe the CSP-process CoopSys3, such that three robots cooperatively transport a box, by replacing the ready-flag with a ready-counter in the CSP-process RoboMng of Fig. 10 and adding the third robot in the CSP-process CoopSys of Fig. 15. However, FDR failed in the model checking for deadlock-freedom of CoopSys 3 due to lack of memory (i.e. state-space explosion) in the MacBook-Pro used in Sect. 4.3. It will be difficult to complete the model checking for CoopSys 3 even by the other model checker or even by using a high-performance computer.

FDR provides a function, named wbisim, for reducing the number of states by folding two or more weaklybisimilar states into one state [5], without changing observational behavior, where weak-bisimilarity is an equivalence relation between two states (i.e. two behaviors). For example, the CSP-process P_RTC35 with 59, 929, 208 states in Fig. 22 can be reduced to W_RTC35 with 3,114 states by the function wbisim, where W_RTC35 is weakly bisimilar to P_RTC35. The CSP-process P_RTC35 in Fig. 22 is the composition of RTC(3) (i.e. RoboCtrlRTC) and RTC(5) (i.e. RaspberryPiMouseRTC), where RTC $(i)$ in Fig. 22 is the CSP-process defined in Fig. 14 and the events in Ch35, which are local events used only between RTC(3) and RTC(5), are hidden and prioritized over tock. The example shows a possibility that larger systems such as CoopSys 3 can be checked if local events are hierarchically hidden and the number of states is reduced for each local structure, but it has to be noted that the reductions by wbisim need non-negligible computation costs. For example, it took more than 10 minutes to reduce P_RTC35 to W_RTC35, which consists of two RTCs with state-reduction, by FDR, while the model checking of CoopSys, which consists of seven RTCs without state-reduction, took about 80 seconds as explained in Sect. 4.3. The functions for state-reduction such

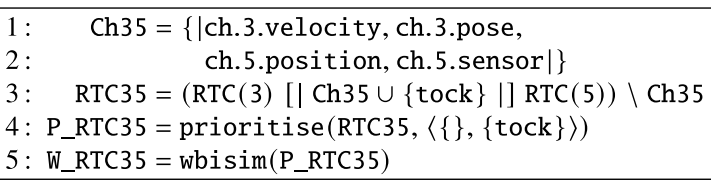

Fig. 22 An example of composition and state-reduction as wbisim are powerful, but they have to be carefully applied.

In order to avoid state-space explosion in model checking, abstraction of values in variables (e.g. expression by a small number of discrete values) are useful as explained in Sect.3.2.3. For example, in the CSP-process RaspberryPiMouseRTC (see Fig. 12), the continuous variable for the position of the real Raspberry-Pi Mouse is expressed by the discrete variable whose range is $\{0,1,2,3\}$, and an abstract technique (namely, non-deterministic choice by internal choice $\Pi$ ) is used for covering the wide-range of the real position. By concretion of values in variables, more accurate behaviors can be formally expressed, but it will easily cause state-space explosion. In model checking, the range of each variable has to be carefully decided.

\section{Implementation of CoopRobo}

In this section, we briefly introduce how to implement CoopRobo on the RT-Middleware (RTM).

RTM is a software platform standard in OMG (Object Management Group) for constructing robot systems by hierarchically connecting RT-Components (RTCs) [12], and OpenRTM-aist [9], [10] is an implementation of RTM. RTM connects RTCs through broadcast channels with ringbuffers according to a configuration-file, in which buffercapacities, overwriting flags, and connections are described. FSM4RTC [11], [13] is an extended-standard of RTC in OMG for supporting implementation of FSMs in RTCs, and is implemented as a library [10] of RTM.

We implemented the FSMs, RoboMng, RoboCtrl, and Client, which are defined in Sect. 2.3, in RTCs with the help of the library FSM4RTC in Python. Although the detail of the Python code is out of scope of this paper, a part of the Python code for the substate Approach in RoboMng is shown in Fig. 23. The first def (line 5) for in_ready in Fig. 23 defines that the flag $r d y$ is set to True when the event

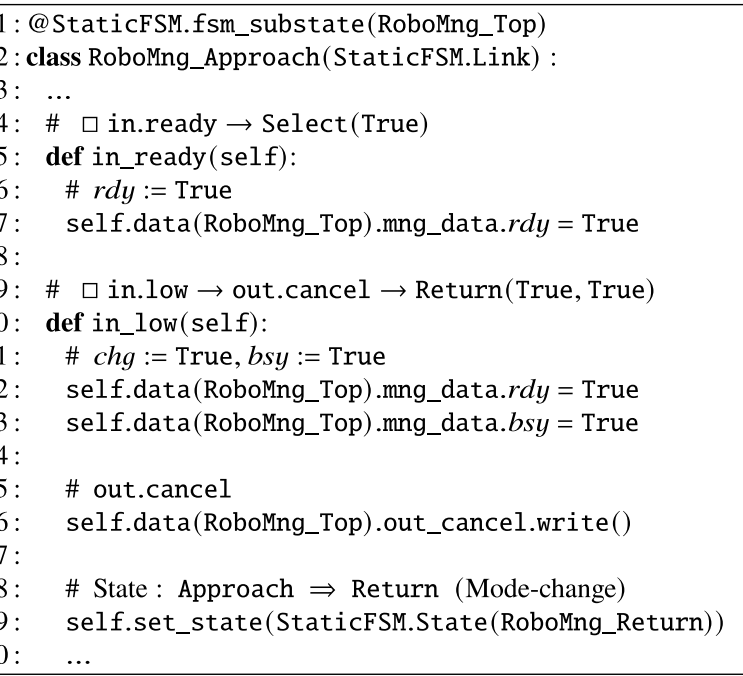

Fig. 23 A part of the Python code for the substate Approach in RoboMng 


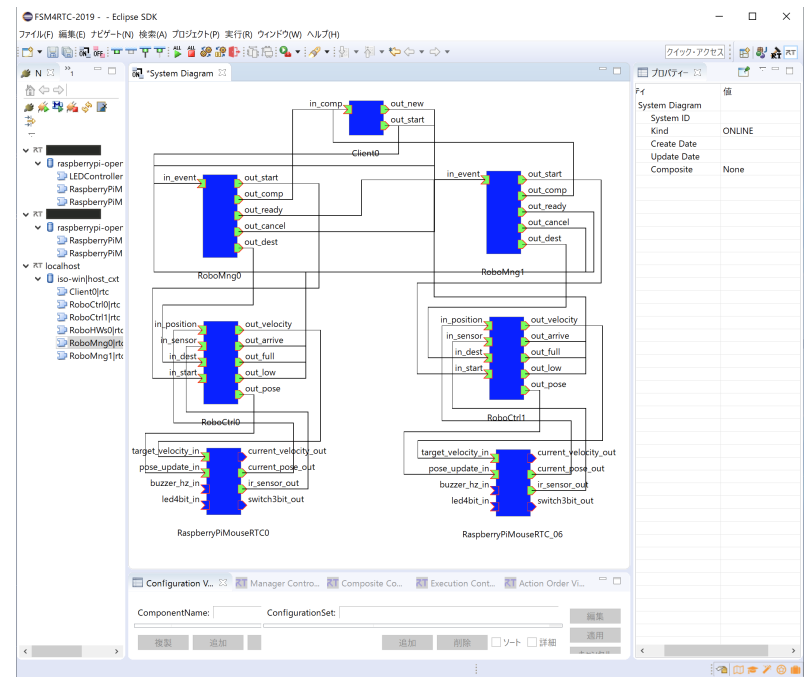

Fig. 24 The graphical connections between RTCs by the RTCBuilder

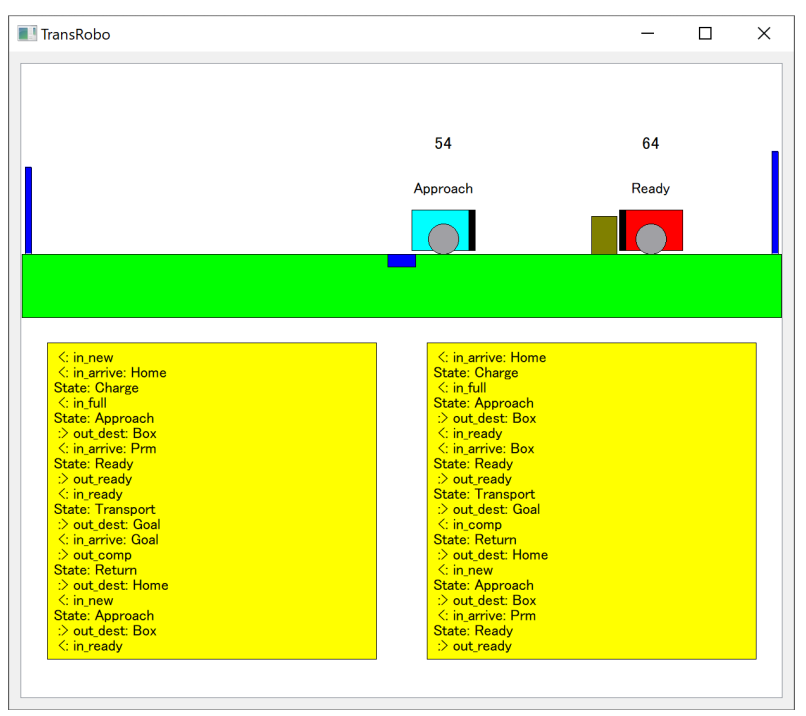

Fig. 25 The simple simulator of CoopSys

ready is received. The second def (line 10) for in_low defines that the flags chg and bsy are set to True, the event cancel is sent, and then the state is changed to the state Return, when the event low is received. As shown in this example, FSMs can be reasonably translated to Python codes in RTCs. The automatic translation tool from FSMs to Python codes is also currently being developed and the prototype of the tool is published in the GitHub [17].

Next, we connected the RTCs by a tool RTCBuilder [9], which provides a graphical editor as shown in Fig. 24, where all the input-events to RoboMng are folded in the input-event in.event of RoboMng, and RaspberryPiMouseRTC is the RTC for Raspberry-Pi Mouse [6] which is published from the GitHub [7]. A demonstrative video of the cooperative transportation by the Raspberry-Pi Mouse is available in the web-site [16] and Fig. 1 is a snapshot of the video.

In addition, we implemented a simple simulator for testing the cooperative robot system CoopSys as shown in Fig. 25. The simulator includes an RTC, named as RoboHWsRTC, which has the same channel-interfaces as the ones of RaspberryPiMouseRTC. The RTC RoboHWsRTC can simulate the behavior of the two robots CoopRobo(1) and CoopRobo(2) with a graphical display to show the positions of the robots, the battery-levels, event-messages passed between robots, and so on. We can easily execute the simulator by replacing the two RTCs of RaspberryPiMouseRTC with RoboHWsRTC.

\section{Related Work}

Luckcuck et al. [1] presented a comprehensive survey on formal specification and verification in robotics. The most related work to ours is RoboChart [18].

RoboChart [18] is a sophisticated robotic system design notation which integrates CSP with a graphical timed state machine notation for defining formal semantics. RoboChart is supported by an Eclipse-based environment, called RoboTool [19], which allows the graphical construction of RoboChart diagrams and can translate them to CSPM for verification by the model checker FDR. RoboChart is very generic and powerful notation for expressing hierarchical timed finite state machines for robots. Our cooperative robot system CoopSys can be also probably expressed in RoboChart even if it is necessary to modify RoboChart in accordance with the communication-style of RTM. However, RoboChart is too generic (powerful) to express our example CoopSys. RoboTool generates a lot of small CSPprocesses which are concurrently or sequentially combined, and it increases the number of states. The number of states can be reduced by state-reduction functions, e.g. diamondreduction, in FDR, but it sometimes takes a lot of time. For example, a case study of transport robots by RoboChart is also published in the web-site [20], but for the verification of the individual transport robot Pusher version 1, the diamondreduction of the CSP-process of the robot was not finished even in 40 hours and crashed in the MacBook-Pro used in Sect. 4.3. The automatic translation to CSP-processes is convenient but sometimes makes it difficult to manually modify the generated CSP-processes.

In this paper, we manually describe the CSP-processes of the cooperative robot system CoopSys as simple as possible, and directly use the model checker FDR for verifying the interactions between robots. Although the formalization of the concurrent-composition of RTCs with FSMs in the communication-style of RTM is error-prone, the CSPprocess for RTM in Fig. 14 is reusable for the other FSMs. The sequential behavior expressed in FSMs (e.g. Fig. 6) can be reasonably translated to CSP-processes (e.g. Fig. 10).

Gjondrekaj et al. [21] presented a formal approach for collective robotic systems and illustrated it through a robotics scenario, in which three robots cooperate to transport an object to a goal area. In the approach, the detail of the scenario is formally modeled with spatial aspects (e.g. positions of robots), temporal aspects (e.g. action execution time), and stochastic aspects (e.g. unfixed action execution 
time) in the formal language StoKLaim. Then, the continuous behavior, which has no discrete mode-change, of the distributed three robots during the cooperative transportation are verified by a statistical model checking algorithm. It is possible to express discrete mode-changes in SтоKLAIM, but hybrid verification for continuous and discrete behaviors require a huge amount of computational power and easily cause state-space explosion. In this paper, we focus only on the discrete controls of mode-changes and abstract the continuous controls to avoid the state-space explosion. For example, it is an interesting future work to verify the continuous controls while the both robots are in the mode Transport by the formal approach of Gjondrekaj et al. [21].

The model checker FDR [5] is used for the formal verification in this paper, while the other formal languages and model checkers can be applied to formal modeling and verification techniques. For example, PAT [22] can exhaustively verify concurrent systems described in TimedCSP [15], which is useful for checking real-time constraint such as deadline or delay. However, verification in TimedCSP requires very higher computation power than the one in CSP with a simple discrete time event tock. In the first step for formal verification of cooperative systems, the pair of CSP and FDR is reasonable.

Instead of model checking, theorem proving can be used for formal verification of software systems. Theorem provers such as Isabelle [23], [24] are generic proofassistant tools and semi-automatically support to describe mathematical proofs by rewriting expressions such as CSPprocesses, which can contain unbounded data-values. For example, RoboChart provides a formalization [25] of a state machine meta-model in Isabelle/UTP to verify robotic systems, although the concurrent composition has not been encoded. Theorem provers are powerful tools for formal verification, but the formal verification-cost of concurrent (cooperative) behaviors often requires a huge amount of manual (not automatic) proofs even though concurrency theories such as CSP have already been encoded on Isabelle/HOL, for example, namely CSP-Prover [26], [27] for semi-automatically proving refinement-relations between CSP-processes.

As explained in the survey [1], various formal approaches have been proposed for supporting the development of cooperative systems, but a comprehensive approach has not been established yet. It is still a challenging work to formally model cooperative systems such as CoopSys given in Sect. 2, and to detect design-errors, for example, leading to the sole transportation explained in Sect. 4.

\section{Conclusion}

Concurrent FSMs (Finite state machines) can express complex behavior of cooperative robots with some discrete modes and event-based cooperation can reduce the communication traffic between robots. The various formal approaches have been proposed for supporting the develop- ment of such cooperative systems, but it is not easy to apply formal approaches because of, for example, low scalability for automatic approaches (e.g. model checkers) and high manual costs for semi-automatic approaches (e.g. theorem provers).

In this paper, we have explained how to formalize cooperative transportation robots with FSMs in the specification language CSP and how to verify the correctness of the behavior by the model checker FDR, where some abstractions of the behavior are applied as explained in Sect. 3.2 to avoid state-space explosion. In this case study, we found several design-errors by FDR, for example, insufficient cancel-notifications corrected in Sect. 4.3. It is hard to detect such design-errors by testing the implementation of the design because failures caused by the errors are rarely observed. It is still costly to formalize and verify cooperative systems, but it is useful for detecting design-errors of cooperative systems, which contain non-deterministic behaviors, and can reduce the costs for implementation and testing.

In fact, we very carefully designed the FSM RoboMng in Fig. 6 and formalized it in CSP in this case study, but the model checker FDR detected the design-errors as explained in Sect. 4.3. Even if we implement the system CoopSys based on the FSM in Fig. 6 without the correction explained in Sect. 4.3, it will almost work well because sole transportation is caused when the two events ready and cancel simultaneously occur. However, the possibility of the sole transportation is not zero. Model checking is one of methods for improving reliability of cooperative robot designs because it exhaustively checks all reachable states in nondeterministic behaviors and then detects assertion-violation.

Reusable formal descriptions such as Figs. 14 and 19 can support the application of formal approaches. It is our future work to construct a library package of such reusable descriptions.

\section{Acknowledgments}

This work was supported by JSPS-KAKENHI Grant Number JP15H02687.

References

[1] M. Luckcuck, M. Farrell, L. Dennis, C. Dixon, and M. Fisher, "Formal Specification and Verification of Autonomous Robotic Systems: A Survey," arXiv:1807.00048, 2019.

[2] C.A.R. Hoare, "Communicating sequential processes," Communications of the ACM, vol.21, no.8, pp.666-677, 1978 .

[3] A.W. Roscoe, The Theory and Practice of Concurrency, Prentice Hall, 1998.

[4] A.W. Roscoe, "Understanding Concurrent Systems," Springer, 2010.

[5] University of Oxford, "FDR4 - The CSP Refinement Checker." https://www.cs.ox.ac.uk/projects/fdr/

[6] RT Corporation, "Raspberry Pi Mouse," https://www.rt-net.jp/ products/raspimouse 3

[7] “The RTC for Raspberry Pi Mouse," https://github.com/rsdlab/ RaspberryPiMouseRTC 
[8] N. Ando, T. Suehiro, K. Kitagaki, T. Kotoku, and W.-K. Yoon, "RT-Middleware: Distributed Component Middleware for RT (Robot Technology)," 2005 IEEE/RSJ International Conference on Intelligent Robots and Systems (IROS2005), pp.3555-3560, 2005.

[9] AIST, "OpenRTM-aist official website," https://openrtm.org/

[10] AIST, "OpenRTM-aist with FSM4RTC in Python," https://github. com/OpenRTM/OpenRTM-aist-Python

[11] N. Ando, "About the standard "FSM4RTC" for state machine components and data ports," SICE SI2016, pp.2584-2587, 2016.

[12] OMG available specification, "Robot Technology Component Specification Version 1.1," formal/2012-09-01. https://www.omg.org/ spec/RTC/

[13] OMG available specification, "Finite State Machine Component For RTC Version 1.0," formal/2016-04-01. https://www.omg.org/ spec/FSM4RTC/

[14] L. Chaimowicz, M.F.M. Campos, and V. Kumar, "Hybrid systems modeling of cooperative robots," Proceedings IEEE International Conference on Robotics and Automation 2003, vol.3, pp.4086-4091, 2003.

[15] S. Schneider, "Concurrent and Real-time systems." Wiley, 2000.

[16] Y. Isobe, "Webpage on Formal Modeling and Verification of Cooperative Transport Robots," https://staff.aist.go.jp/y-isobe/ csp-cooprobo/

[17] AIST, "The system development tools for OpenRTM-aist," https://github.com/OpenRTM/OpenRTP-aist

[18] A. Miyazawa, P. Ribeiro, W. Li, A. Cavalcanti, J. Timmis, and J. Woodcock, "RoboChart: modelling and verification of the functional behaviour of robotic applications," Software \& Systems Modeling, volume 18, Springer, pp.3097-3149, 2019.

[19] University of York, "RoboTool: RoboChart Tool," https://www.cs. york.ac.uk/robostar/robotool/

[20] P. Ribeiro, W. Li, A.L.C. Cavalcanti, and A. Sampaio, "Transport: a case study of RoboTool," https://www.cs.york.ac.uk/robostar/ case_studies/transport/transport.html

[21] E. Gjondrekaj, M. Loreti, R. Pugliese, F. Tiezzi, C. Pinciroli, M. Brambilla, M. Birattari, and M. Dorigo, "Towards a Formal Verification Methodology for Collective Robotic Systems," International Conference on Formal Engineering Methods (ICFEM), pp.54-70, LNCS 7635, Springer, 2012.

[22] National University of Singapre, "PAT: Process Analysis Toolkit," http://www.comp.nus.edu.sg/pat/

[23] T. Nipkow, L.C. Paulson, and M. Wenzel, "Isabelle/HOL - A Proof Assistant for Higher-Order Logic,” LNCS 2283, Springer, 2002.

[24] University of Cambridge and Technische Universität München, "Isabelle: A Generic Theorem Prover," https://isabelle.in.tum.de/

[25] S. Foster, J. Baxter, A. Cavalcanti, A. Miyazawa, and J. Woodcock, "Automating Verification of State Machines with Reactive Designs and Isabelle/UTP," International Conference on Formal Aspects of Component Software (FACS), LNCS 11222, Springer, pp.137-155, 2018.

[26] Y. Isobe and M. Roggenbach, "A Generic Theorem Prover of CSP Refinement," International Conference on Tools and Algorithms for the Construction and Analysis of Systems (TACAS), LNCS 3440, Springer, pp.108-123, 2005.

[27] Y. Isobe and M. Roggenbach, "Webpage on CSP-Prover," https://staff.aist.go.jp/y-isobe/CSP-Prover/CSP-Prover.html

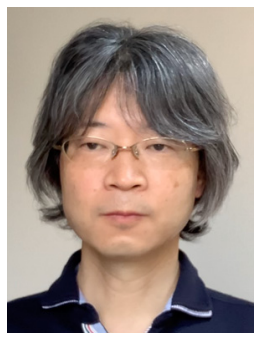

Yoshinao Isobe received his BEng and MEng degrees in Electrical Engineering from Shibaura Institute of Technology in 1990 and 1992 respectively. In 1992, he joined Electrotechnical Laboratory, MITI. He received his $\mathrm{Ph}$.D. degree from Shizuoka University in 2001. He was a visiting researcher of the University of Wales, Swansea for one year in 2003. He was also a special appointment associate professor in the National Institute of Informatics in 20122014. He is currently a senior researcher in the National Institute of Advanced Industrial Science and Technology. His research interests include formal verification of concurrent systems. He is a member of IEICE, JSSST, and IPSJ.

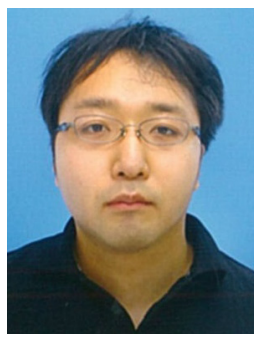

Nobuhiko Miyamoto received his BEng and MEng degrees from Ritsumeikan University in 2011 and 2013 respectively. He is a doctoral student of the Graduate School of System Design, Tokyo Metropolitan University, since 2017. He is also a software engineer of the Software Platform Research Team, Industrial CPS Research Center in AIST. He is interested in research on robot middleware, software architecture, formal methods, educational robotics, robot control, and mobile robot mechanism. He is member of RSJ, SICE, and IEEJ.

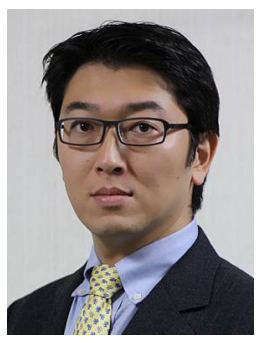

Noriaki Ando received MEng and Ph.D. degrees from the University of Tokyo, in 1999 and 2002, respectively. He joined National Institute of Advanced Industrial Science and Technology (AIST) in 2003, and is currently the leader of Software Platform Research Team, Industrial CPS Research Center in AIST. His research interests include robot system integration, teleoperation, robot middleware, and its standardization. He has also proposed and standardized a specification of the robotic technology component (RTC) and implemented OpenRTM-aist based on the RTC standard. $\mathrm{He}$ is a member of IEEE, SICE, RSJ and JSME.

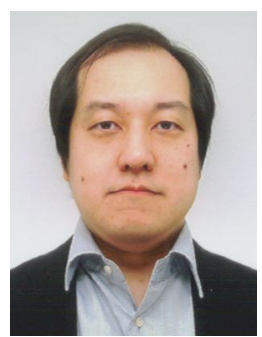

Yutaka Oiwa received his Ph.D. degree in Computer Science from the University of Tokyo in 2005. He joined National Institute of Advanced Industrial Science and Technology (AIST) in 2005, and is currently the leader of Software Quality Assurance Research Team, Cyber Physical Security Research Center in AIST. He is a visiting professor in Nara Institute of Science and Technology. His research interest covers programming languages, software implementation, software engineering, network protocol and security, IoT architecture, and safety of computer systems using artificial intelligence. He is a member of ACM, IPSJ and JSSST. 